\title{
The pyroptosome: a supramolecular assembly of ASC dimers mediating inflammatory cell death via caspase-1 activation
}

\author{
T Fernandes-Alnemri, J Wu, J-W Yu, P Datta, B Miller, W Jankowski, S Rosenberg, J Zhang and ES Alnemri
}

Pyroptosis is a caspase-1-dependent inflammatory form of cell death. The adapter protein ASC binds directly to caspase-1 and is critical for caspase- 1 activation in response to a broad range of stimuli. To elucidate the mechanism of activation of caspase-1 by ASC and its exact role in macrophage pyroptosis, we performed time-lapse confocal bioimaging analysis on human THP-1 macrophages stably expressing an ASC-GFP fusion protein. We show that stimulation of these cells with several proinflammatory stimuli trigger the formation of a large supramolecular assembly of ASC, termed here pyroptosome. Only one distinct pyroptosome in each stimulated cell is formed, which rapidly recruits and activates caspase-1 resulting in pyroptosis and the release of the intracellular proinflammatory cytokines. The pyroptosome is largely composed of oligomerized ASC dimers. Dimerization of ASC is driven by subphysiological concentrations of potassium as in vitro incubation of purified recombinant ASC in the presence of subphysiological concentrations of potassium induces the assembly of a functional pyroptosome. Furthermore, stimulation of potassium efflux in THP-1 cells with potassium-depleting agents induces formation of the pyroptosome, while increasing potassium concentrations in the culture medium or pharmacological inhibition of this efflux inhibits its assembly. Our results establish that macrophage pyroptosis is mediated by a unique pyroptosome, distinct from the inflammasome.

Cell Death and Differentiation (2007) 14, 1590-1604; doi:10.1038/sj.cdd.4402194; published online 29 June 2007

Macrophages and monocytes express a battery of plasma membrane-associated toll-like receptors (TLRs) and cytosolic Nod/NACHT-LRR (NLR) proteins that recognize a vast array of pathogen-associated molecular patterns (PAMPs) and non-pathogen-associated danger signals. ${ }^{1-3}$ In response to these stimuli, macrophages and monocytes produce pro-IL$1 \beta$ and pro-IL-18 and activate their caspase-1. The active caspase-1 processes the inactive pro-IL-1 $\beta$ and pro-IL-18 to produce the active cytokines IL-1 $\beta$ and IL-18, respectively, which are then released into the extracellular space. Active IL-1 $\beta$ and IL-18 are potent mediators of inflammation that stimulate fever, recruitment and activation of immune cells, and production of secondary cytokines. ${ }^{4}$

Caspase- 1 is activated by cytosolic complexes termed inflammasomes. ${ }^{5,6}$ In the past few years a number of inflammasome complexes have been identified including ICE-protease activating factor (IPAF), NALP1, NALP2, cryopyrin/NALP3, and pyrin inflammasomes. ${ }^{5,7-10}$ IPAF and NALP1, both members of the NLR family, associates directly with caspase- 1 via CARD-CARD interactions and promote its activation by inducing dimerization of the inactive monomeric procaspase- $1 .{ }^{9,11}$ On the other hand, the NLR proteins NALP2 and cryopyrin/NALP3, and the TRIM-family member pyrin do not associate directly with pro-caspase- 1 and require the adapter protein ASC to recruit and activate caspase-1.7,10

Although little is known about the mechanisms of activation of the inflammasomes, recent studies revealed that different inflammasomes are activated by different signals. Infection of macrophages with intracellular bacterial pathogens such as Salmonella typhimurium or cytosolic exposure to purified flagellin protein from these pathogens, have been shown to activate specifically the IPAF inflammasome (reviewed in Mariathasan $S^{6}$ ), whereas exposure to anthrax lethal toxin or MDP have been shown to activate the NALP1 inflammasome. ${ }^{11,12}$ Interestingly, the cryopyrin inflammasome is activated by a broad range of signals including infection with intracellular bacteria Listeria monocytogenes and Staphylococcus aureus, TLR agonists plus potassium-depleting agents such as ATP, nigericin or maitotoxin, danger signal monosodium urate (MSU), antiviral compounds R837 and R847, bacterial RNA and viral double-stranded RNA. ${ }^{13-17}$ The mechanism by which these diverse signals converge to

Department of Biochemistry and Molecular Biology, Center for Apoptosis Research, Kimmel Cancer Institute, Thomas Jefferson University, Philadelphia, PA, USA ${ }^{*}$ Corresponding author: E Alnemri, Department of Biochemistry and Molecular Biology, Center for Apoptosis Research, Thomas Jefferson University, $233 \mathrm{~S}$. 10th St, Philadelphia, PA 19107, USA. Tel: 215503 4632; Fax: 215923 1098; E-mail: e_alnemri@lac.jci.tju.edu

Keywords: pyroptosis; ASC; pyroptosome; inflammasome; caspase-1; potassium

Abbreviations: CARD, caspase-associated recruitment domain; PYD, pyrin domain; NOD, nucleotide-binding oligomerization domain; LRR, leucine-rich repeat; NLR, NACHT-LRR; IL, interleukin; NACHT, NTPase-domain named after NAIP, CIITA, HET-E and TP1; NALP, NACHT, LRR and Pyrin domain containing; LPS, lipopolysaccharide; TLR, toll-like receptors; ASC, apoptosis-associated speck-like protein containing a CARD; IPAF, ICE-protease activating factor; PAMP, pathogenassociated molecular patterns; TRIM, tripartite motif; MDP, muramyl dipeptide; MSU, monosodium urate; GFP, green fluorescent protein; WT, wild type; RT, reverse transcription; FMK, fluoromethyl ketone; PCR, polymerase chain reaction; GST, glutathione S-transferase; FSL-1, fibroblast-stimulating lipopeptide-1; LDH, lactate dehydrogenase; SDS-PAGE, sodium dodecyl sulfate-polyacrylamide gel electrophoresis; DSS, disuccinimidyl suberate; PMA, phorbol 12-myristate 13-acetate; SAT, staphylococcus aureus $\alpha$-toxin; TEA, tetraethylammonium; ELISA, enzyme-linked immunosorbent assay

Received 19.4.07; revised 22.5.07; accepted 01.6.07; Edited by G Melino; published online 29.6.07 
activate the cryopyrin inflammasome is not clear, but it is likely that they all activate a common physiological response that triggers cryopyrin inflammasome activation.

ASC is a 22-kDa adapter protein with an N-terminal PYD and a C-terminal CARD. ${ }^{18}$ It links the PYD-containing NLR family member to procaspase-1, using its PYD to interact with the PYD of the NLRs and its CARD to interact with the CARD of procaspase-1. ${ }^{5,8}$ Gene-targeted deletion of ASC in mice revealed that ASC is essential for activation of caspase- 1 and generation of mature $\mathrm{IL}-1 \beta$ by almost all stimuli known to induce IL-1 $\beta$ generation, suggesting that $\mathrm{ASC}$ is a key downstream effector of caspase-1 activation. ${ }^{13-15,17,19-21}$ How ASC induces caspase-1 activation is not clear, but it is currently believed that PYD-containing NLR family members such as cryopyrin assemble an inflammasome complex with ASC, which in turn recruits and activates caspase-1.,7 However, there is so far no direct in vivo evidence that this ternary complex is indeed assembled in response to proinflammatory stimuli.

Recently, it has been demonstrated that activation of caspase-1 not only leads to inflammation, but in certain instances causes an inflammatory form of cell death called pyroptosis. ${ }^{22-25}$ Pyroptosis was initially observed in macrophages infected with the intracellular bacteria S.typhimurium, ${ }^{26}$ but was later found to occur in response to infection with a number of other intracellular bacterial and viral pathogens (reviewed in Swanson $\mathrm{MS}^{22}$ and Fink $\mathrm{SL}^{24}$ ). An inflammatory form of cell death not caused by pathogenic infection, but similar to pyroptosis, was also described in human THP-1 macrophage cell line and murine macrophages in response to different stimuli including bacterial toxins, lipoproteins and LPS plus ATP. ${ }^{27-31}$ Since all these stimuli have been recently shown to activate caspase-1 in macrophages, it is likely that this infection-independent inflammatory form of cell death or pyroptosis is also dependent on caspase-1 activation.

Little is known about the mechanism by which caspase-1 can be specifically activated to induce pyroptosis. Given that ASC deficiency completely abrogated caspase- 1 activation in response to $S$. typhimurium infection, ${ }^{19}$ it is clear that ASC plays a critical role in $S$. typhimurium-induced pyroptotic cell death. However, the role of ASC in infection-independent cell death is not clear. In this report, we investigated the role of ASC in non-infection-induced macrophage pyroptosis, and the mechanism by which ASC activates caspase- 1 to induce this form of cell death. Our results show for the first time that ASC forms a single 1-2 $\mu \mathrm{m}$ inflammasome-independent supramolecular assembly in each macrophage cell in response to stimulation with proinflammatory stimuli. This ASC supramolecular assembly is formed in response to potassium depletion and is composed of oligomerized ASC dimers. Given that the ASC supramolecular assembly can recruit and activate caspase-1 to induce pyroptosis, we refer to it as the ASC pyroptosome.

\section{Results}

LPS induces formation of an ASC supramolecular assembly in THP-1 macrophages. Crude LPS preparations from Gram-negative bacteria or bacterial lipoproteins have been shown to induce caspase-1 activation and a pyroptosis-like cell death in human THP-1 macrophages. ${ }^{29-32}$ However, the mechanism by which caspase- 1 is activated in these cells, and the role of ASC were not determined. As a first step to characterize the role of ASC in this model of inflammatory cell death, we generated a THP-1 macrophage cell line that stably expresses physiological amounts of an ASC-GFP fusion protein (Figure 1a and c). In unstimulated cells, ASC-GFP was evenly distributed in the cytoplasm and nucleus. However, after stimulation with crude Escherichia coli LPS for $1 \mathrm{~h}$, the entire ASC-GFP fluorescence accumulated as distinct bright clusters or oligomers in the cytoplasm of $\sim 30 \%$ of the treated cells (Figure 1a). These oligomers are large and measured about $2 \mu \mathrm{m}$ in diameter and only one oligomer is formed in each cell (Figure 1b). We designated these ASC oligomers pyroptosomes, since all cells containing these oligomers showed morphological and biochemical characteristics of pyroptosis (see below).

Quantitative analysis of the number of cells with ASC pyroptosomes revealed that the number of cells containing these pyroptosomes increased in a time- and dose-dependent fashion after stimulation with LPS (Figure 1d). At moderate LPS concentrations, more than $90 \%$ of pyroptosome formation occurred within the first $2 \mathrm{~h}$ after LPS treatments. Formation of the ASC pyroptosomes did not require new protein synthesis as preincubation of cells with the protein synthesis inhibitor cycloheximide did not inhibit their formation in response to LPS treatment (Figure 1f).

Since the cytotoxic effect of crude LPS preparations is attributed to contamination with bacterial lipoproteins, ${ }^{31}$ we tested whether the synthetic bacterial lipopeptide analogs Pam3CSK4 and FSL-1, which have been shown to induce cell death in THP-1 cells, ${ }^{31}$ could also induce formation of the ASC pyroptosome. Treatment of the THP-1-ASC-GFP cells with these lipopeptides also induced a time-dependent formation of ASC pyroptosomes (Figure 1e). These results thus indicate that bacterial lipoproteins in crude LPS preparations are likely responsible for induction of the ASC pyroptosome. Consistent with this conclusion, stimulation of the THP-1-ASC-GFP cells with ultrapure LPS induced very little ASC pyroptosomes, compared to crude LPS (data not shown). In addition, treatment of these cells with MDP, another common contaminant of LPS, did not induce ASC pyroptosome formation (data not shown).

LPS and bacterial Pam3CSK4 or FSL-1 lipopeptides have been shown recently to induce caspase- 1 activation and IL-1 $\beta$ secretion from mouse bone marrow macrophages independent of the cryopyrin inflammasome. ${ }^{13}$ To investigate if signals that activate the cryopyrin inflammasome could also induce the formation of the ASC pyroptosome, we treated THP-1-ASC-GFP cells with the danger signal MSU or the antiviral compound R837, both of which require cryopyrin to activate caspase-1. ${ }^{13,15}$ As shown in Figure 1a and e, MSU and R837 were also able to induce ASC pyroptosome formation in a dose- and time-dependent manner. Combined, these results indicate that formation of the ASC pyroptosome can be induced by both cryopyrin and non-cryopyrindependent signals. 
a


b
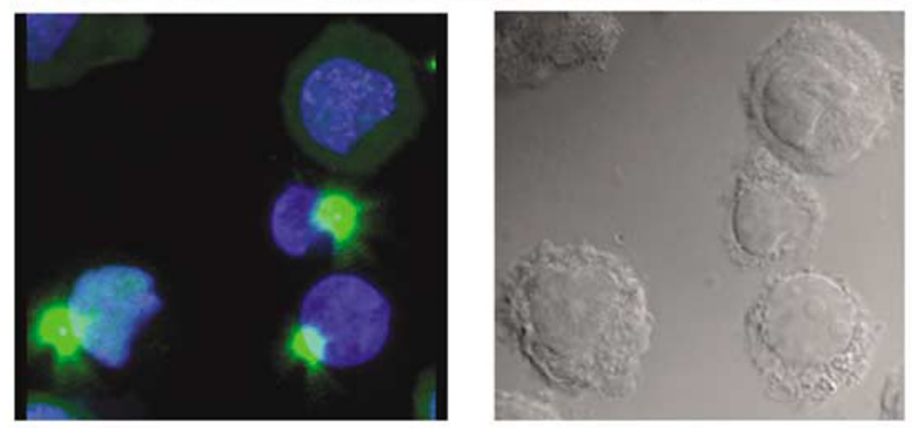

C
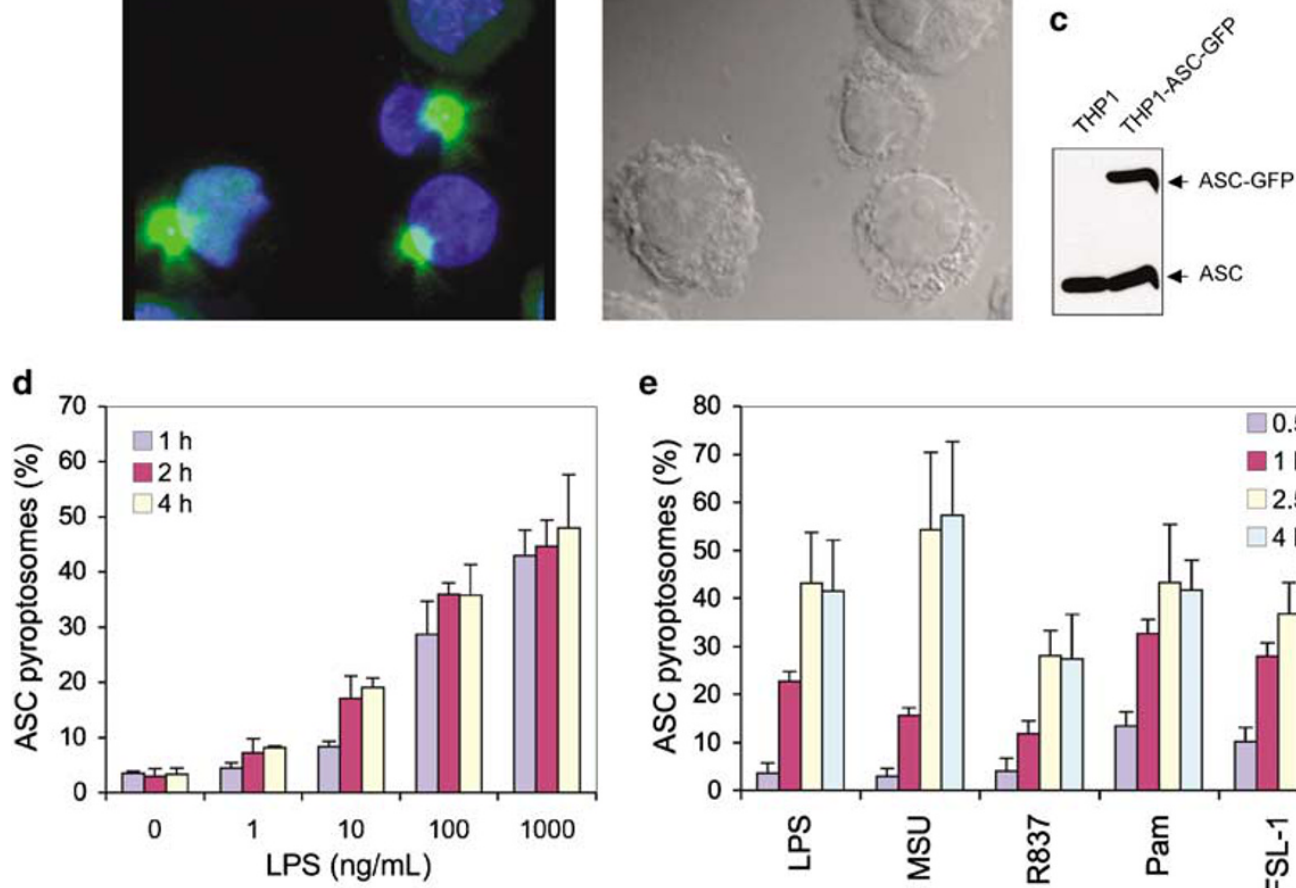

e

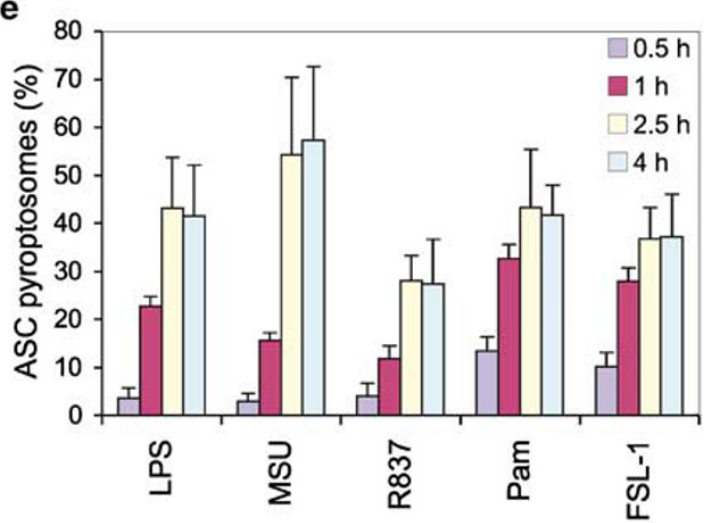

f

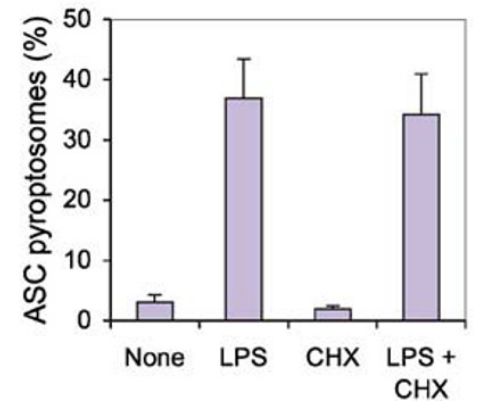

Figure 1 Proinflammatory stimuli induce formation of ASC pyroptosome in THP-1 cells. (a) Live PMA-primed THP-1-ASC-GFP cells were left untreated (c, left micrograph) or treated with LPS $(1 \mu \mathrm{g} / \mathrm{ml})$ (middle micrograph) or MSU $(100 \mu \mathrm{g} / \mathrm{ml})$ (right micrograph) for $60 \mathrm{~min}$ and observed by fluorescence microscopy $(\times 20$ magnification). (b) PMA-primed THP-1-ASC-GFP cells seeded on glass cover slips were treated with crude LPS for 60 min and then fixed and stained with DAPI. Cells were then observed and photographed by fluorescence confocal microscopy ( $\times 63$ magnification). Notice the uniformly distributed green fluorescent ASC-GFP in one cell that did not form ASC pyroptosome (top half of left micrograph) and the large perinuclear green fluorescent ASC pyroptosomes formed in three other cells (bottom half of left micrograph). The right micrograph shows a phase contrast confocal micrograph of the same cells in the left micrograph. (c) Western blot analysis of lysates from parental THP1 or stable THP-1-ASC-GFP cells with anti-ASC antibody. Notice that the expression level of the ASC-GFP fusion protein (upper band) in the THP-1-ASC-GFP cells is comparable to the expression level of endogenous ASC (lower band). (d) THP-1-ASC-GFP cells were pretreated with zVAD-FMK for 30 min and then treated with increasing amounts of crude LPS for the indicated periods of time. The percentages of cells containing ASC pyroptosomes were calculated as described under the 'Materials and Methods'. (e) THP-1-ASC-GFP cells were treated as in (d) with LPS $(1.0 \mu \mathrm{g} / \mathrm{ml}), \mathrm{MSU}(100 \mu \mathrm{g} / \mathrm{ml})$, R837 $(5.0 \mu \mathrm{g} / \mathrm{ml})$ Pam3CSK4 $(1.0 \mu \mathrm{g} / \mathrm{ml})$ or FSL-1 (1.0 $\mu \mathrm{g} / \mathrm{ml})$ for the indicated periods of time. The percentages of cells containing ASC pyroptosomes were determined as in (d). (f) THP-1-ASC-GFP cells were preincubated with cycloheximide $(10 \mu \mathrm{g} / \mathrm{ml})$ for $30 \mathrm{~min}$ and then treated with LPS $(1.0 \mu \mathrm{g} / \mathrm{ml})$ for an additional $1 \mathrm{~h}$. The percentages of cells containing ASC pyroptosomes were determined as in (d) 
Formation of the ASC pyroptosome is rapid and precedes cell death. To observe the dynamics of assembly of the ASC pyroptosome, we performed timelapse confocal imaging on LPS-stimulated THP-1-ASC-GFP cells. These experiments revealed that the entire process from the start until the complete assembly of the whole ASC pyroptosome in a single cell takes less than $3 \mathrm{~min}$ (Figure $2 \mathrm{a}$, first three panels; Supplementary Movies 1-3). Within seconds after ASC pyroptosome formation, the plasma membrane ruptures releasing cellular contents and membrane vesicles in the culture supernatants. The plasma membrane then appears to reseal rapidly and starts to swell to form a balloon-shaped vesicle around the nucleus, which also undergoes dramatic rounding and condensation. The mitochondria also lose membrane potential and release their contents as evidenced by the concurrent loss of the mitochondrial vital stain mitotracker. These phenotypic features were also observed in the parental THP-1 macrophages after treatment with LPS (data not shown), which indicates that oligomerization of ASC causes cell death in THP-1 macrophages.

To determine whether the LPS-induced pyroptosome formation and cell death is dependent on caspase activation, cells were pretreated with the pancaspase inhibitor zVADFMK and then stimulated with LPS. Pretreatment with zVADFMK blocked all the morphological features of cell death, but did not affect ASC pyroptosome formation in response to LPS treatment (Figure $2 \mathrm{~b}$ and Supplementary Movies 4 and 5). These data indicate that LPS-induced cell death is caspasedependent. In contrast, pyroptosome formation per se is not dependent on caspase activation. The morphological features of this form of cell death are clearly a combination of apoptosis (condensation of the nucleus and loss of mitochondrial membrane integrity) and oncosis (plasma membrane swelling). Since this form of cell death is associated with caspase-1 activation and secretion of inflammatory cytokines (see below), we will refer to it as pyroptosis. ${ }^{24}$

Plasma membrane swelling and rupture triggered by formation of the ASC pyroptosome might be a mechanism by which dying macrophages release their cellular contents of inflammatory cytokines such as IL-1 $\beta$. Indeed, stimulation of the parental THP-1 cells with different proinflammatory agents induced concomitant cell death as measured by LDH release and $\mathrm{IL}-1 \beta$ release in the culture media (Figure $2 \mathrm{c}$ and $\mathrm{d}$ ). Similar results were obtained with THP-1-ASC-GFP cells (data not shown). Collectively, our data indicate that proinflammatory stimuli cause a pyroptotic cell death in THP-1 macrophages by inducing the formation of an ASC pyroptosome. This form of cell death is responsible for the release of the mature IL- $1 \beta$ cytokine from activated macrophages.

Caspase-1 is the apical caspase in the macrophage pyroptotic cell death pathway. The rapid activation of pyroptotic cell death after formation of the ASC pyroptosome and the ability of the pan caspase inhibitor to block this form of cell death, but not the formation of the ASC pyroptosome, suggests that the ASC pyroptosome is responsible for activation of a cellular caspase that causes cell death. Considering that ASC can only associate with procaspase1,8 it is likely that activation of caspase-1 by the ASC pyroptosome is responsible for the pyroptotic cell death observed in THP-1 cells. To test this hypothesis, we devised a biochemical procedure to isolate the ASC pyroptosome from the parental THP-1 macrophages after LPS stimulation. Since the diameter of the ASC-GFP pyroptosome in the THP-1-ASC-GFP cells is $\sim 2 \mu \mathrm{m}$, we estimated that the diameter of the untagged endogenous ASC pyroptosome in the parental THP-1 cells could be in the range of $\sim 1 \mu \mathrm{m}$. Therefore, it is possible to separate the ASC pyroptosome from nuclei by a combination of membrane filtration through a 5-micron-filter to remove nuclei, and centrifugation at relatively low speed (5000 r.p.m.) to pellet the ASC pyroptosomes. We performed this procedure on control and LPS-stimulated THP-1 cells that were previously preincubated with zVAD-FMK to prevent cell death and trap the apical caspase on the ASC pyroptosome. The resulting cell-free pellets were then subjected to chemical crosslinking with the non-cleavable protein crosslinking agent Disuccinimidyl suberate (DSS) to determine the oligomeric state of ASC in the control and LPS-stimulated cells. As shown in Figure 3a (upper panel), ASC pyroptosomes were predominantly present in the pellets from the LPS-stimulated cells (fifth lane) compared to the control unstimulated cells (second lane), which contained very small amount of ASC. Chemical crosslinking of the isolated pellets yielded crosslinked ASC oligomers in the pellets from the LPStreated cells (sixth lane), but not from the control untreated cells (third lane). The ASC dimer was the major crosslinked species, indicating that the ASC pyroptosome is composed of oligomerized ASC dimers. The ASC pyroptosomes were associated with unprocessed and processed caspase-1 p35 fragment as determined by Western blotting with a specific caspase-1 antibody (Figure 3, lower panel), indicating that the ASC pyroptosomes recruit and activate caspase- 1 after LPS-stimulation of THP-1 cells.

To provide additional evidence that caspase- 1 is the apical caspase in the pyroptotic cell death pathway triggered by formation of the ASC pyroptosome, we generated stable HEK293 cells expressing physiological amounts of ASC-GFP protein alone (293-ASC-GFP cells), or ASC-GFP and procaspase-1 together (293-C1-ASC-GFP). Stimulation with PMA-induced formation of ASC pyroptosomes in both cell lines (Supplementary Figure 1). However, only cells expressing both ASC-GFP and procaspase-1 (293-C1-ASC-GFP) showed morphological features of pyroptosis. The 293-ASCGFP cells, which do not express caspase-1 remained viable even after formation of the ASC pyroptosome (Supplementary Figure 1, middle panel). Control HEK293 cells, stably transfected with procaspase-1 and GFP alone (293-C1GFP), but ASC, was also not sensitive to PMA-induced pyroptosis. These results indicate that the ASC pyroptosome induces pyroptosis by activating caspase-1 not only in macrophages, but also in caspase-1-expressing cell lines like 293-C1-ASC-GFP cells.

To investigate further the role of caspase-1 in the pyroptotic process, we isolated bone marrow macrophages from WT and caspase $-1^{-1-}$ mice and stimulated them with LPS for $3 \mathrm{~h}$ followed by ATP for $1 \mathrm{~h}$. Unlike human THP-1 cells, stimulation of primary mouse macrophages with LPS alone does not induce robust caspase- 1 activation and requires an ATP 
a
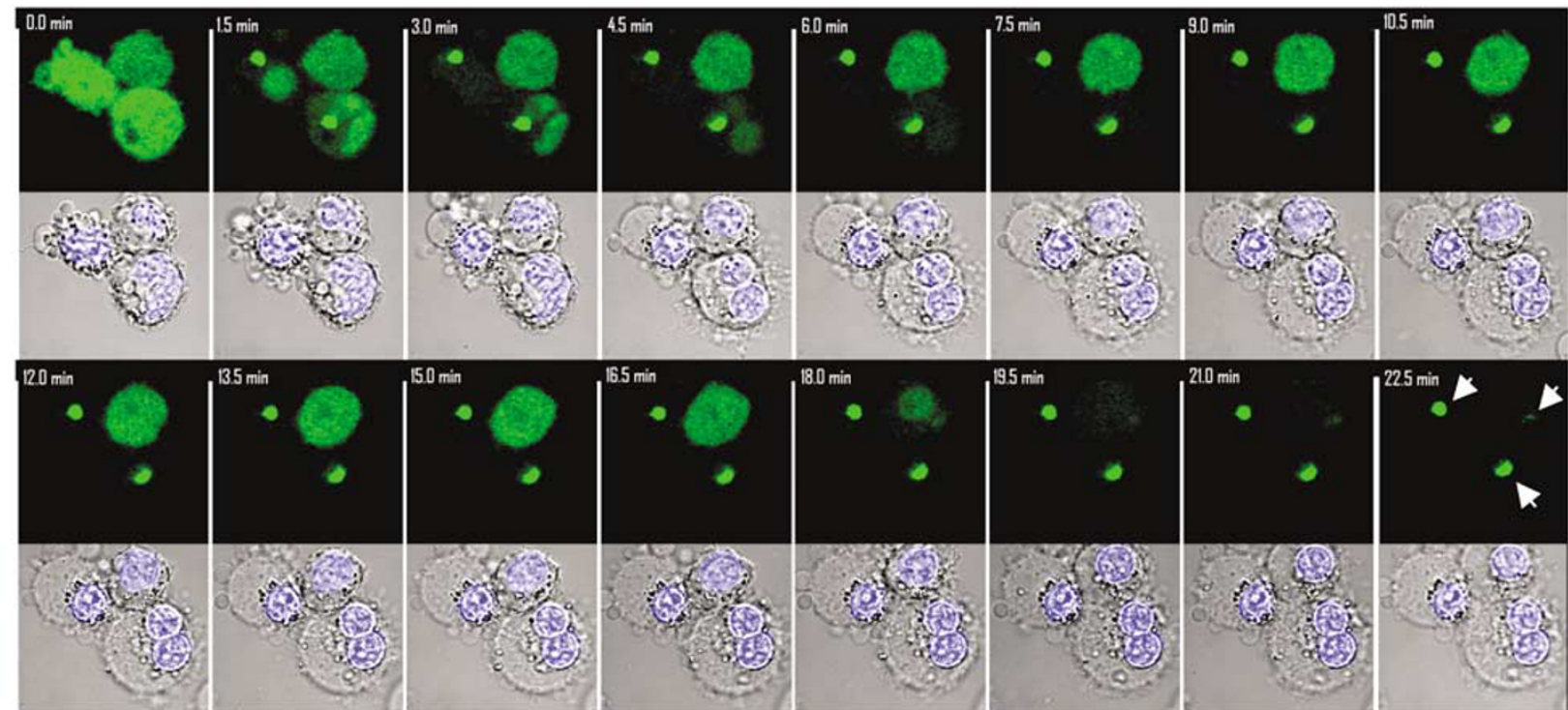

b
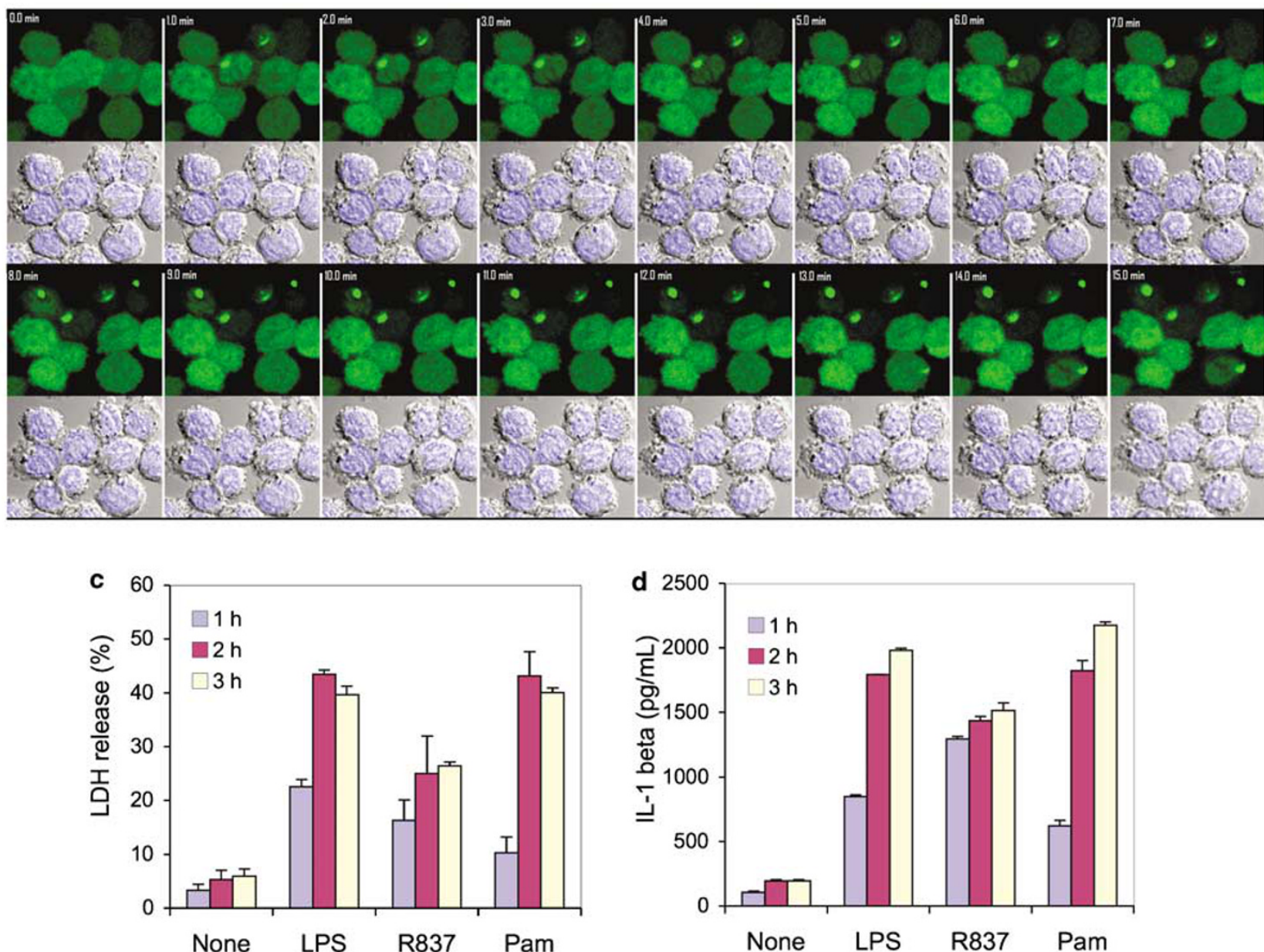

Figure 2 Formation of the ASC pyroptosome causes cell death in THP-1 cells. (a and $\mathbf{b})$ Time-series confocal images ( $\times 63$ magnification) of crude LPS-stimulated THP1-ASC-GFP cells in the absence (a, 1.5 min images are shown) or presence of ZVAD-FMK $(70 \mu \mathrm{M})(\mathbf{b}, 1.0$ min images are shown). The arrows in the last image point to ASC pyroptosomes. Time-lapse movies showing the assembly of the ASC pyroptosome in the absence or presence of ZVAD-FMK can be seen in the 'Supplementary Movies 1-5'. (c and d) Pyroptosome-induced pyroptotosis causes the release of intracellular LDH and IL-1 $\beta$. PMA-primed parental THP-1 or THP-1-ASC-GFP cells were pretreated for $1 \mathrm{~h}$ with ultrapure LPS to induce pro-IL-1 $\beta$ and then treated with crude LPS $(1.0 \mu \mathrm{g} / \mathrm{ml})$, R837 $(10 \mu \mathrm{g} / \mathrm{ml})$ or Pam3CSK4 $(0.5 \mu \mathrm{g} / \mathrm{ml})$ for the indicated periods of time. LDH release into the culture medium is shown as a percentage of LDH release by detergent (c). IL-1 $\beta$ release into the culture media was determined by ELISA (d) 
a

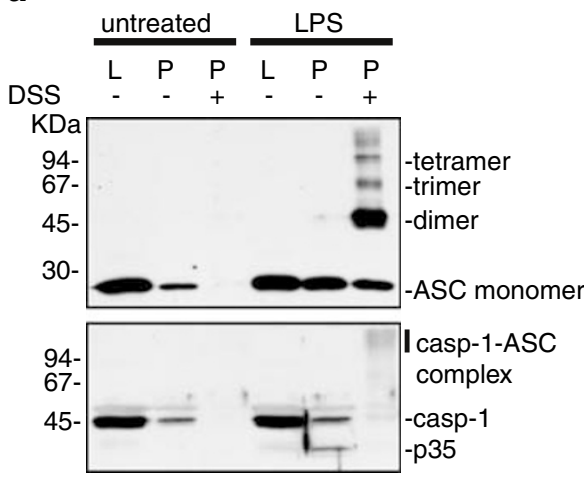

b

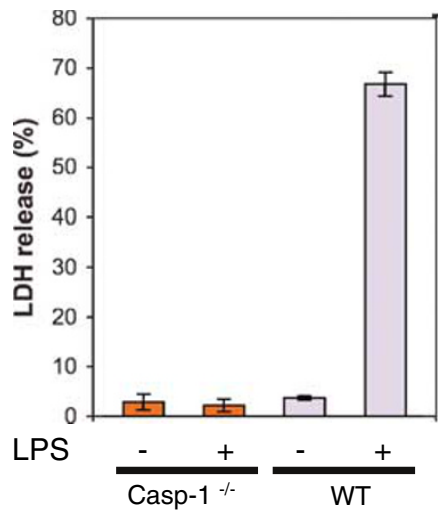

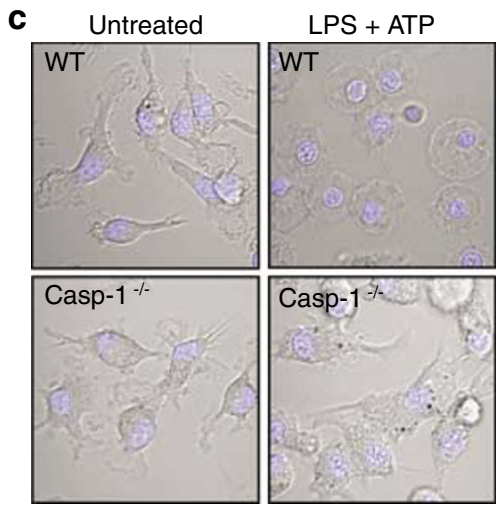

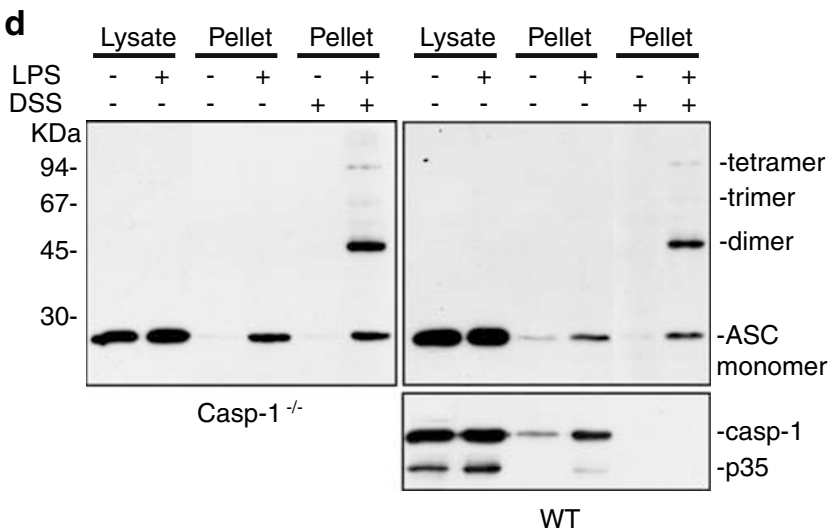

Figure 3 Caspase-1 is the apical caspase in pyroptosis and inflammation. (a) THP-1 cells were left untreated or treated with LPS $(1.0 \mu \mathrm{g} / \mathrm{ml})$ for $3 \mathrm{~h}$ in the presence of zVAD-FMK and then lysed. The ASC pyroptosomes present in the lysates were pelleted by centrifugation at 5000 r.p.m. as described under 'Materials and Methods'. Two-third of the pellets (P) was incubated with DSS for $30 \mathrm{~min}$ (third and sixth lanes) and the remaining one-third was left untreated (second and fifth lanes). The lysates (L) and pellets (P) were then fractionated by SDS-PAGE and Western blotted with anti-ASC (upper panel) or anti-caspase-1 (lower panel) antibodies. (b) Bone marrow macrophages from WT or caspase- $1^{-1-}$ mice were treated with LPS $(1.0 \mu \mathrm{g} / \mathrm{ml})$ for $3 \mathrm{~h}$ followed by ATP $(4 \mathrm{mM})$ for $1 \mathrm{~h}$, and the LDH release into the culture medium was determined as described under 'Materials and Methods'. (c) Confocal micrographs of bone marrow macrophages from WT or caspase $-1^{-1-}$ mice treated with LPS plus ATP. Notice the pyroptotic phenotype in the LPS plus ATP-treated WT (top right panel), but not the caspase- $1^{-1-}$ (bottom right panel) cells. (d) ASC pyroptosomes were isolated from WT or caspase- $1^{-1-}$ bone marrow macrophages after treatment with LPS plus ATP. The ASC pyroptosomes were isolated as in (a). The lysates and pellets were then fractionated by SDS-PAGE and Western blotted with anti-ASC (upper panel) or anti-caspase-1 (lower panel) antibodies

stimulus to activate caspase-1..$^{14}$ Stimulation with LPS plus ATP-induced cell death in WT macrophages but not in caspase- $1^{-1-}$ cells as revealed by LDH release (Figure $3 b$ ). The morphological features of cell death in these cells resembled THP-1 cell death, with nuclear condensation and plasma membrane swelling (Figure 3c), indicating that these cells die by pyroptosis, which is mediated by caspase- 1 activation.

As with LPS-stimulated THP-1 cells, LPS plus ATP. stimulated WT and caspase-1-deficient mouse macrophages contained ASC pyroptosomes, as revealed by chemicalcrosslinking (Figure $3 d$, sixth and 12th lanes). These results indicate that stimulation with LPS plus ATP induces formation of ASC pyroptosomes in both WT and caspase- $1^{-1-}$ macrophages. However, formation of ASC pyroptosome alone does not induce cell death, but requires the presence of procaspase- 1 to produce active caspase-1, which in turn processes its physiological substrate pro-IL-1 $\beta$ and cause cell death to release the active IL-1 $\beta$ cytokine from the macrophages.
The ASC pyroptosome is a potent caspase-1 activation platform. To provide direct biochemical evidence that the ASC pyroptosome is indeed capable of activating procaspase-1, we purified ASC pyroptosomes from LPSstimulated THP-1 cells. The purified ASC pyroptosomes were then incubated with recombinant WT or active-site mutant (C285A) procaspase-1. As shown in Figure $4 \mathrm{a}$, purified ASC pyroptosomes induced activation of the WT caspase-1, but not the C285A caspase-1 mutant. The activated WT caspase- 1 was able to process pro-IL-1 $\beta$ to the mature IL-1 $\beta$ cytokine, indicating that purified ASC pyroptosome is responsible for activating caspase- 1 and generation of mature IL-1 $\beta$ during pyroptosis.

The kinetics of assembly of the ASC pyroptosome in LPSstimulated THP-1-ASC-GFP cells and the fact that only one pyroptosome per cell is formed suggests that the assembly process is driven by self-association of the ASC monomers to form dimers, which then oligomerize to form a large ASC pyroptosome. To test if this process can be recapitulated 
a

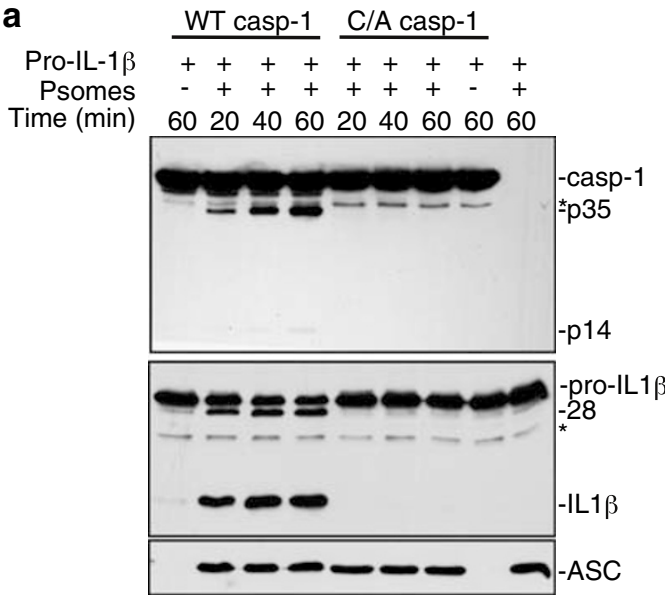

C

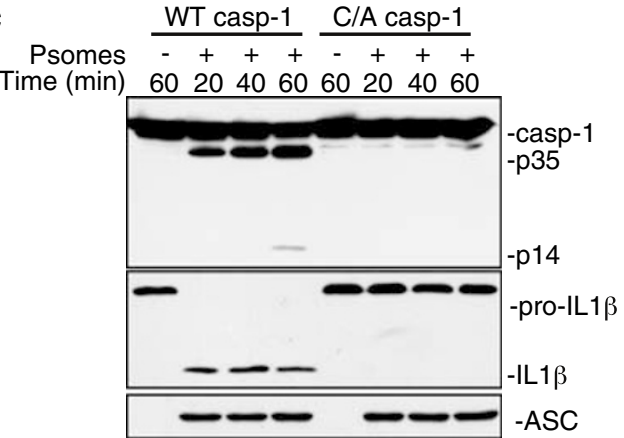

b
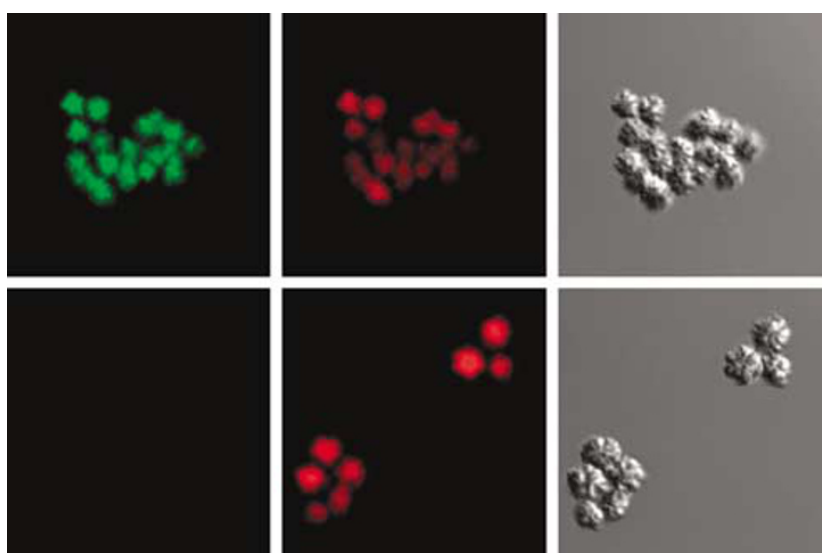

d
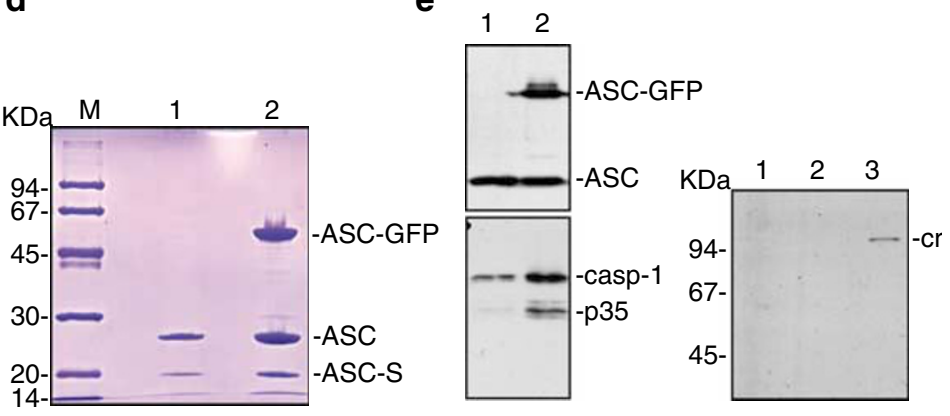

Figure 4 The ASC pyroptosome is the molecular platform responsible for recruitment and activation of caspase-1. (a) ASC pyroptosomes purified from LPS-stimulated THP-1-ASC-GFP cells were incubated with flag-tagged WT or active-site mutant (C/A) procaspase-1 together with pro-IL-1 $\beta$ at $37^{\circ} \mathrm{C}$ for 20,40 , or 60 min as indicated. The reaction products were then analyzed by SDS-PAGE and Western blotting with anti-flag (upper panel) or anti-IL-1 $\beta$ (middle panel) antibodies. Notice that only WT caspase-1 (lanes 1-4), but not active-site mutant caspase-1 (C/A) (lanes 5-8), can be activated by ASC pyroptosomes. The asterisks indicate a non-specific band. (b) Confocal micrographs ( $\times 63$ magnification) of ASC-GFP pyroptosomes purified from THP-1-ASC-GFP cell lysates (upper panels), or endogenous ASC pyroptosomes from THP-1 cell lysates (lower panels). The ASC pyroptosomes were isolated in the presence of rhodamine-labeled zVAD-fmk to label the associated caspase-1 (middle red panels). Phase contrast (right panels). Notice the highly organized star-shaped structure of the ASC pyroptosome. (c) Same as in (a) but using in vitro assembled and purified ASC pyroptosomes from THP-1 lysate. (d) The purified ASC pyroptosomes shown in (b) were fractionated by SDS-PAGE and stained with Coomassie Blue. Lane 1: endogenous ASC pyroptosomes purified from THP-1 lysate. Lane 2: ASC pyroptosomes purified from THP-1-ASC-GFP lysate. Notice the presence of endogenous ASC (lane 2, middle band) in the ASC-GFP pyroptosome preparation. The ASC-GFP (lane 2, top band) migrates slightly above the $45 \mathrm{kDa}$ marker. The bottom band in lanes 1 and 2 is an endogenous short isoform of ASC (ASC-S). (e) Western blots of purified in vitro assembled ASC pyroptosomes from THP-1 cell lysates (first lanes), or purified ASC pyroptosomes from LPS-stimulated THP-1-ASC-GFP cells (second lanes). The pyroptosomes were isolated in the presence of ZVAD-FMK to trap the activated caspase-1 on the pyroptosomes. The blots were probed with anti-ASC (left upper panel, exposure time 15s), anti-caspase-1 (left lower panel, exposure time $30 \mathrm{~s}$ ) or anti-cryopyrin (right panel, exposure time $30 \mathrm{~min}$ ) antibodies. The third lane in the cryopyrin blot is a positive cryopyrin-containing lysates control from a stable 293 cells expressing cryopyrin

in vitro by incubation of lysates from THP-1 cells at $37^{\circ} \mathrm{C}$, we prepared S100 cell lysates from THP-1-ASC-GFP and the parental THP-1 cells in a hypotonic buffer and incubated the resulting lysates on ice or at $37^{\circ} \mathrm{C}$ for $30 \mathrm{~min}$ in the presence of rhodamine-tagged zVAD-FMK (red-zVAD) to label and trapactivated caspase-1 on the ASC pyroptosome. The lysates were then centrifuged at 5000 r.p.m. to pellet the ASC pyroptosomes, and the pellets containing ASC pyroptosomes were further purified by centrifugation over a $40 \%$ percoll cushion. Examination of the purified pellets by fluorescent confocal microscopy revealed that incubation at $37^{\circ} \mathrm{C}$ induced formation of ASC pyroptosomes in these lysates (Figure 4b). These ASC pyroptosomes bound red-zVAD-FMK indicating that they contain activated caspase-1. The shape of these ASC pyroptosomes were similar to those formed in vivo in LPS-stimulated THP-1-ASC-GFP cells, but were larger in size and measured $\sim 3 \mu \mathrm{m}$ in diameter. The in vitro assembled ASC pyroptosomes from both THP-1 and THP-1-ASC-GFP cells have star-shaped crystal-like (quasicrystal) structure, indicating that they are not merely aggregates of ASC. Interestingly, the ASC-GFP pyroptosomes (upper panels) have sharper spikes than the ASC pyroptosomes (lower panels), possibly because of the presence of GFP at the $C$ terminus of ASC. No ASC pyroptosomes were seen in lysates incubated on ice (not shown). These results indicate that ASC pyroptosomes can form spontaneously in cell-free lysates by mere incubation at $37^{\circ} \mathrm{C}$. These results also explain previous observations, which showed that caspase- 1 is spontaneously activated in hypotonic THP-1 lysates after incubation at $37^{\circ} \mathrm{C}^{5}$

To determine whether the in vitro assembled ASC pyroptosomes could activate caspase-1, we incubated purified ASC 
pyroptosomes from $37^{\circ} \mathrm{C}$-activated THP-1 lysates with recombinant WT or active-site mutant (C285A) procaspase1. As expected, the purified ASC pyroptosomes activated WT procaspase-1, but not the active-site mutant (C285A) procaspase-1 (Figure 4c, upper panel). The active WT caspase- 1 was able to process pro-IL-1 $\beta$ to the mature $\mathrm{IL}-1 \beta$ cytokine (Figure $4 \mathrm{c}$, lower panel). Combined, these results indicate that the ASC pyroptosome is indeed responsible for activation of caspase-1.

To determine the composition of the ASC pyroptosome, we subjected large preparations of purified pyroptosomes from either THP-1 (Figure 4d, lane 1) or THP-1-ASC-GFP (Figure 4d, lane 2) cells to SDS-PAGE and Coomassie staining. The pyroptosome preparation from THP-1 cells contained two distinct bands migrating as 19 and $25 \mathrm{kDa}$ species (Figure 4d, lane 1), which were identified by Western blotting and Mass Spectroscopy to be full length ASC (25 kDa band) and a short isoform of ASC (19 kDa band). Similarly, the pyroptosome preparation from THP-1-ASC-GFP cells contained the 25 and $19 \mathrm{kDa}$ ASC species together with the ASC-GFP fusion protein, which migrates as a $\sim 50 \mathrm{kDa}$ band (Figure 4d, lane 2). No high-molecular-weight species in the range of $90-110 \mathrm{kDa}$ corresponding to cryopyrin or other related family members were detected in these preparations. Very faint bands ranging in sizes between 30 and $50 \mathrm{kDa}$ were also detected in these preparations. These bands correspond to proform and processed caspase-1 as determined by Western blotting and mass spectroscopy, which detected only ASC and caspase-1 (Figure 4e, left panels), but not cryopyrin (Figure 4e, right panel), in these preparations. These results demonstrate clearly that the ASC pyroptosome is composed largely of oligomerized ASC dimers and a small amount of caspase-1. The absence of cryopyrin or other NLRs from the purified pyroptosome cannot be attributed to dissociation of these proteins during purification, since the pyroptosome purification process, which is a gentle few step process did not dissociate caspase- 1 from the pyroptosome. Furthermore, quantitative estimates of the cellular concentrations of ASC $(\sim 1.4 \mu \mathrm{g} / \mathrm{mg}$ total cytosolic proteins) and cryopyrin ( $\sim 15 \mathrm{pg} / \mathrm{mg}$ total cytosolic proteins) in THP-1 as measured by quantitative immunoblot analysis with specific ASC and cryopyrin antibodies revealed that the molar ratio of ASC to cryopyrin in THP-1 cells is $\sim 500-1$. Therefore, it is unlikely that the endogenous ASC pyroptosome would contain equimolar amounts of ASC and cryopyrin, since almost all cellular ASC assembles into one pyroptosome in each stimulated THP-1 cell (Figure 2a).

The ASC pyroptosome is formed by self-association of the pyrin domain of ASC. ASC contains two domains, an $\mathrm{N}$-terminal pyrin domain (PYD) and a C-terminal CARD domain. The $\mathrm{N}$-terminal PYD has been shown to mediate self-association of ASC. ${ }^{33}$ To determine if the PYD of ASC is responsible for formation of the ASC pyroptosome, we introduced a point mutation (K26A) in the PYD of ASC, which has been previously shown to prevent self-association of the isolated PYD of ASC. ${ }^{33}$ The WT and mutant ASC proteins were then stably expressed in 293-caspase-1 cells. Incubation of lysates from the WT ASC expressing cells, but not the $\mathrm{K} 26 \mathrm{~A}$ ASC mutant at $37^{\circ} \mathrm{C}$ induced caspase- 1 activation (Figure 5a, lane 2). Chemical crosslinking of lysates from these cells revealed that only the WT ASC but not the K26A mutant was able to form ASC pyroptosomes (Figure 5b, lane 2).

To provide additional evidence that the PYD is responsible for assembly of the ASC pyroptosome, whereas the CARD domain is needed to recruit procaspase-1, we generated a chimeric ASC molecule, which contains ASC PYD (residues $1-100$ ) followed by the CARD domain of Apaf-1 (Figure 5c), which interacts with the CARD domain of procaspase-9. This protein was expressed and purified from bacteria and then assembled into pyroptosomes (see below). Incubation of the chimeric ASC pyroptosomes with procaspase-9 resulted in activation of procaspase-9 (Figure $5 d$, lane 3). As expected, the chimeric ASC pyroptosome was able to activate a chimeric procaspase-1, which contains the CARD domain of procaspase- 9 instead of its original CARD, but not WT procaspase-1 (Figure 5e, left panels). Together, these results indicate that ASC forms pyroptosomes by self-association of its PYD, whereas the CARD domain recruits and activates procaspase-1.

The assembly of the ASC pyroptosome is mediated by potassium depletion. Unrelated and diverse agents such as LPS, MSU, R837, Pam3CSK4 and FSL-1 can induce the formation of the ASC pyroptosome. Despite their distinct mechanisms of action, these agents might stimulate a common downstream event that triggers formation of the ASC pyroptosome. One possibility is that these agents alter the physiological milieu of the cell, which is sensed either by a molecule upstream of ASC (i.e., cryopyrin) or by ASC itself, triggering ASC oligomerization. Potassium efflux is a common event induced by a broad range of stimuli and has been shown to play an important role in cell death, caspase activation and IL-1 $\beta$ processing. ${ }^{14,27,28,34,35}$ Given that depletion of intracellular potassium by bacterial toxins has been shown to induce pyroptotic cell death and $\mathrm{IL}-1 \beta$ release from THP-1 cells, ${ }^{27}$ we asked whether intracellular potassium depletion induces formation of the ASC pyroptosome in macrophages. To answer this question, we examined the effect of inhibiting potassium efflux by increasing the extracellular potassium concentration to 30 or $60 \mathrm{~mm}$. As shown in Figure $6 \mathrm{a}$, inhibition of potassium efflux by high extracellular potassium concentrations inhibited LPS-induced formation of the ASC pyroptosome in these cells. High extracellular potassium also blocked pyroptotosis and IL-1 $\beta$ secretion from THP-1 cells as measured by LDH release and ELISA, respectively (Figure $6 \mathrm{~b}$ and $\mathrm{c}$ ). Moreover, high extracellular potassium inhibited Pam3CSK4 and R837-induced ASC pyroptosome formation, pyroptosis and IL-1 $\beta$ secretion from THP-1 cells (data not shown).

The potassium channel blocker tetraethylammonium (TEA) has been shown to inhibit IL-1 $\beta$ secretion from human monocytes. ${ }^{34}$ To provide additional evidence that the formation of ASC pyroptosome is dependent on intracellular potassium depletion, we incubated the THP-1-ASC-GFP cells in the presence of TEA. As expected, inhibition of potassium efflux by TEA also decreased LPS-induced ASC pyroptosome formation at a concentration as low as $2 \mathrm{~mm}$ (Figure 6a). 




C



b

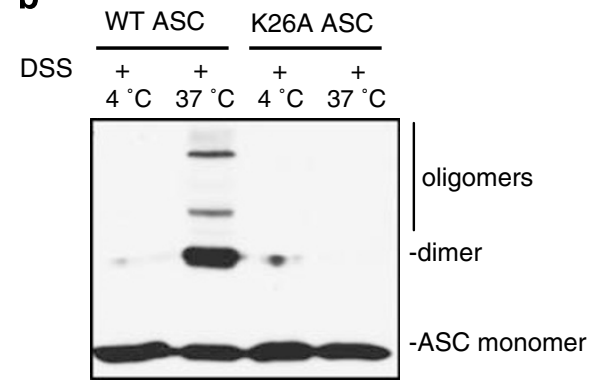

d

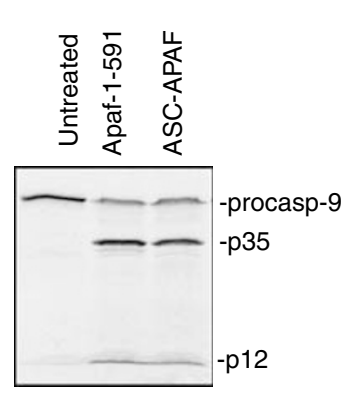

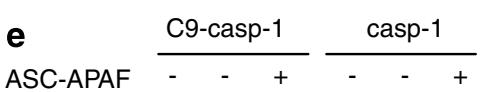

$\begin{array}{lllllll}\left({ }^{\circ} \mathrm{C}\right) & 4 & 37 & 37 & 4 & 37 & 37\end{array}$

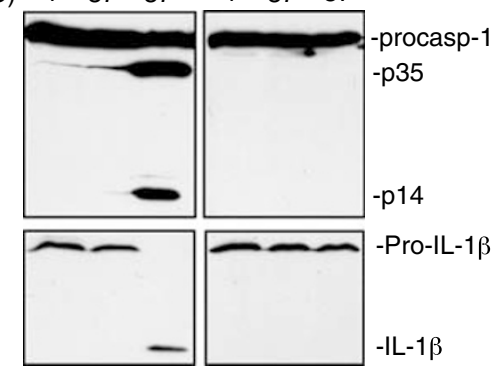

Figure 5 The pyrin domain of ASC mediates formation of the ASC pyroptosome. (a) Lysates from stable 293T cells (10 $\mu \mathrm{g} / \mu$ l) expressing flag-tagged procaspase-1 and either WT or K26A mutant ASC were activated by incubation at $37^{\circ} \mathrm{C}$ or left at $4^{\circ} \mathrm{C}$ for $1 \mathrm{~h}$ as indicated. The lysates were then analyzed by SDS-PAGE and Western blotted with anti-flag (upper panel) or anti-ASC (lower panel) antibodies. (b) The lysates described in (a) were incubated at 4 or $37^{\circ} \mathrm{C}$ for 30 min with the crosslinking agent DSS as indicated. The lysates were then analyzed by SDS-PAGE and Western blotted with anti-ASC antibody. (c) A schematic illustration of the chimeric ASC-APAF which contains the CARD of Apaf- 1 at its $C$ terminus instead of its original CARD, and the C9-procaspase-1, which contains the CARD of procaspase- 9 at its $\mathrm{N}$ terminus instead of its original CARD. (d) Bacterially produced chimeric ASC-APAF pyroptosomes or Apaf-1-591 were incubated with ${ }^{35} \mathrm{~S}$-labeled procaspases-9 at $37^{\circ} \mathrm{C}$ for $1 \mathrm{~h}$ as indicated. The reaction products were then analyzed by SDS-PAGE and autoradiography. Notice that both ASC-APAF and Apaf-1-591 can activate procaspase-9. (e) Bacterially produced chimeric ASC-APAF pyroptosomes were incubated with flag-tagged C9-procaspase-1 chimera (left panels) or WT procaspase- 1 (right panels) together with pro-IL-1 $\beta$ at 4 or $37^{\circ} \mathrm{C}$ for $1 \mathrm{~h}$ as indicated. The reaction products were then analyzed by SDS-PAGE and Western blotting with anti-flag (upper panels) or anti-IL-1 $\beta$ (lower panels) antibodies

To provide more direct evidence that potassium depletion induces formation of the ASC pyroptosome in THP-1-ASCGFP cells, we stimulated these cells with Staphylococcus aureus $\alpha$-toxin (SAT). SAT selectively permeabilizes the plasma membrane for monovalent ions and has been previously shown to decrease dramatically intracellular potassium concentrations to below $50 \mathrm{mM}$ in THP-1 cells. ${ }^{27}$ Treatment of THP-1-ASC-GFP cells with SAT induced ASC pyroptosome formation in more than $65 \%$ of the cells (Figure 6d). Consistent with the critical role of potassium, treatment of THP-1-ASC-GFP with SAT in the presence of $60 \mathrm{~mm}$ extracellular potassium completely inhibited ASC pyroptosome formation.

To rule out the possibility that other bacterial contaminants in the SAT preparation is responsible for activation of the ASC pyroptosome, we examined the effect of digitonin, a mild nonionic detergent used to permeabilize plasma membrane, on THP-1-ASC-GFP cells (Figure 6e). Treatment of these cells with low concentration of digitonin- $(10 \mu \mathrm{g} / \mathrm{ml})$ induced robust formation of the ASC pyroptosome (Figure 6e). The effect of digitonin was also inhibited by high extracellular potassium (Figure 6e). Together, our results indicate that depletion of intracellular potassium by different agents with distinct mechanisms of actions triggers formation of the ASC pyroptosome.
Subphysiological concentrations of potassium induce ASC oligomerization and enhances recruitment and activation of procaspase-1 by the ASC pyroptosome. How does low intracellular potassium concentration induce formation of the ASC pyroptosome? Based on our observations that the PYD of ASC mediates its selfassociation to form the ASC pyroptosome (Figure 5), it is possible that low potassium concentrations favor selfassociation of the ASC PYD. To test this possibility, we examined the effect of different concentrations of potassium on self-assembly of the ASC pyroptosome in vitro. THP-1 S100 extracts were stimulated by incubation at $37^{\circ} \mathrm{C}$ in the presence of increasing concentrations of potassium for $30 \mathrm{~min}$ and the assembled ASC pyroptosomes were isolated by low-speed centrifugation (Figure 7a, upper panel). As anticipated, no ASC pyroptosomes were found at high or near physiological potassium concentration (fifth and sixth lanes). In contrast, ASC pyroptosomes were found at potassium concentrations below $120 \mathrm{~mm}$ with maximum amount at $30 \mathrm{~mm}$ (lanes 1-4). This experiment demonstrates that indeed, low potassium concentrations favor the assembly of ASC pyroptosomes, whereas physiological concentrations of potassium (150 mM) block assembly in vitro (Figure $7 \mathrm{a}$, upper panel).

To study further the effect of potassium on ASC oligomerization and caspase-1 activation, we incubated THP-1 S100 
a

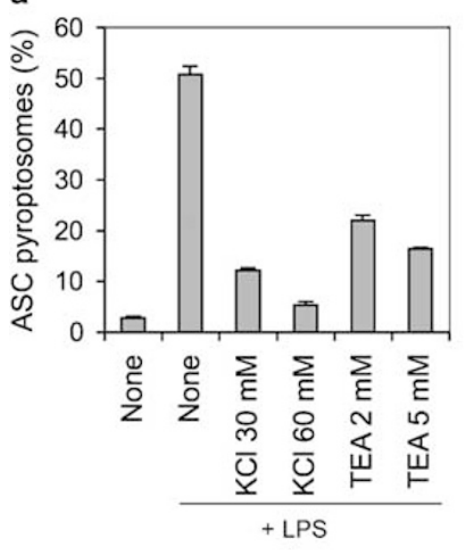

b

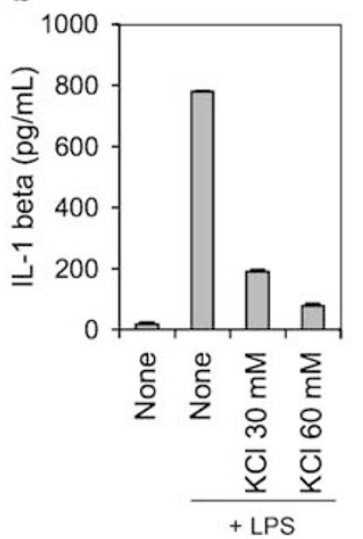

C



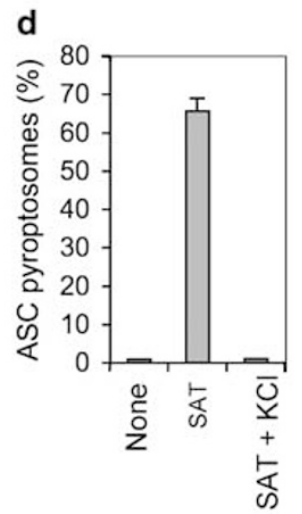



Figure 6 Potassium depletion triggers formation of the ASC pyroptosome in vivo. (a) THP-1-ASC-GFP cells were treated with crude LPS (1 $\mu \mathrm{g} / \mathrm{ml})$ in the absence or presence of the indicated concentrations (mM) of $\mathrm{KCl}$, or the potassium channel blocker TEA for $2 \mathrm{~h}$. The percentages of cells containing ASC-GFP pyroptosomes were determined as in Figure 1d. (b and c) THP-1 cells were treated with crude LPS $(1 \mu \mathrm{g} / \mathrm{ml})$ in the absence or presence of the indicated concentrations of KCl for the indicated periods of time. LDH release into the culture medium is shown as a percentage of LDH release by detergent (c). IL-1 $\beta$ release into the culture media was determined by ELISA (b). (d and e) THP-1-ASC-GFP cells were treated with SAT $(10 \mu \mathrm{g} / \mathrm{ml})$ or digitonin $(10 \mu \mathrm{g} / \mathrm{ml})$ in the absence or presence of the of $\mathrm{KCl}(60 \mathrm{mM})$ as indicated and the percentages of cells containing ASC-GFP pyroptosomes were determined as in Figure 1d

lysates at $37^{\circ} \mathrm{C}$ in the presence of increasing potassium concentrations and then determined the status of caspase-1 in the lysates by Western blotting (Figure 7b). Consistent with the above findings, the results of this experiment show clearly that physiological concentrations of potassium inhibit in vitro caspase-1 activation. Chemical crosslinking of the THP-1 lysates with DSS during incubation at $37^{\circ} \mathrm{C}$ revealed that the potassium-mediated inhibition of caspase- 1 activation is caused by a direct inhibitory effect of potassium on selfoligomerization of ASC (Figure 7c).

Potassium depletion might not only be important for ASC oligomerization, but it could also be important for recruitment and activation of procaspase- 1 by the ASC pyroptosome. To examine the effect of varying potassium concentrations on the recruitment of procaspase- 1 to the ASC pyroptosome, we incubated lysates containing an active site mutant procaspase-1 C285A with purified pre-formed ASC pyroptosomes in the presence of different potassium concentrations. The complexes were then isolated by low-speed centrifugation. As shown in Figure 7d, increasing potassium concentrations inhibited recruitment of procaspase- 1 to the pyroptosome in a dose-dependent manner with maximum inhibition observed at
$150 \mathrm{~mm} \mathrm{KCl}$. Consistent with this result, increasing potassium concentrations also inhibited in a dose-dependent manner, the activation of WT procaspase- 1 in 293 lysates by purified ASC pyroptosomes (Figure 7e, left panels). Similar results were obtained when purified ASC pyroptosomes were incubated with purified procaspase-1 (Figure 7e, right panels). Together, these results indicate that potassium depletion plays a critical role in macrophage pyroptosis by inducing the formation of the ASC pyroptosome and subsequently enhancing the recruitment and activation of caspase-1. Thus, we predict that in the absence of a potassium efflux, procaspase1 cannot be fully activated.

In vitro assembly of the ASC pyroptosome using purified recombinant ASC. We next addressed the question of whether assembly of the ASC pyroptosome could be mediated by an indirect effect of potassium depletion on a molecule upstream of ASC (i.e., cryopyrin), which facilitates ASC oligomerization, or is a direct effect on ASC itself. We purified bacterially expressed ASC to complete homogeneity in a buffer containing physiological concentration of potassium (150 mM). In this buffer, ASC was 
a
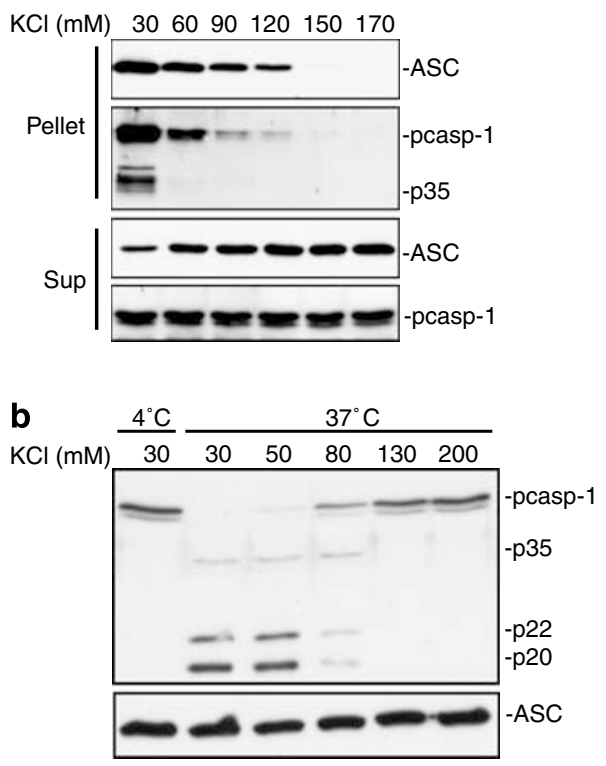

C

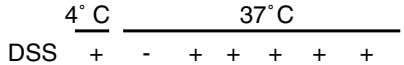

$\mathrm{KCl}(\mathrm{mM}) \quad 30 \quad 30 \quad 3050 \quad 80130200$

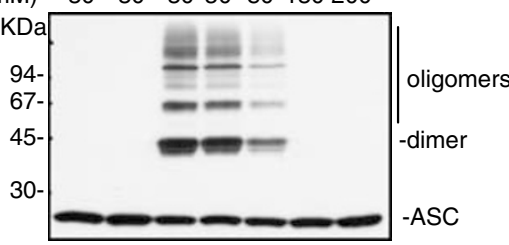

d

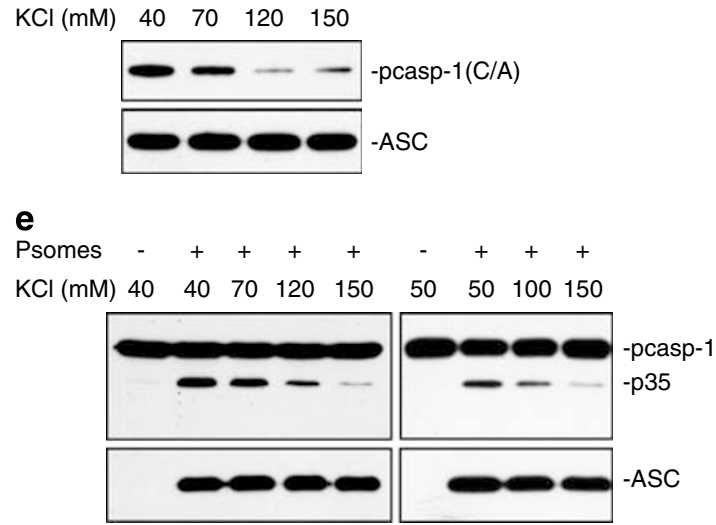

Figure 7 Effect of potassium on pyroptosome assembly in vitro. (a) ASC pyroptosomes were assembled in vitro by incubation of THP-1 S100 extracts $(10 \mu \mathrm{g} / \mu \mathrm{l})$ at $37^{\circ} \mathrm{C}$ in the presence of increasing concentrations of $\mathrm{KCl}$. The reaction mixtures were centrifuged at 5000 r.p.m., and the resulting pellets, which contain the assembled pyroptosomes, and the remaining supernatants were then fractionated by SDS-PAGE followed by Western blotting with anti-ASC or anti-caspase-1 antibodies. The ASC blot (first panel from the top, pellet) was exposed for $2 \mathrm{~min}$. The caspase- 1 blot (second panel from the top, pellet) was exposed for $3 \mathrm{~h}$ to detect caspase-1. The supernatant blots (third and fourth panels from the top) were exposed for $2 \mathrm{~min}$. (b and $\mathbf{c}$ ) Lysates from THP-1 cells $(10 \mu \mathrm{g} / \mu \mathrm{l})$ were incubated at $4^{\circ} \mathrm{C}$ or activated at $37^{\circ} \mathrm{C}$ in the presence of the indicated concentrations of $\mathrm{KCl}$ together with (b) or without (c) the crosslinking agent DSS $(4 \mu \mathrm{M})$. The lysates were analyzed by Western blotting with anti-caspase-1 (b) or anti-ASC (c) antibodies. (d) Purified preformed ASC pyroptosomes were incubated with 293 lysates containing inactive flag-procaspase-1 mutant (C285A) in the presence of the indicated potassium concentrations at $37^{\circ} \mathrm{C}$ for $1 \mathrm{~h}$. The ASC pyroptosomes were then pelleted by centrifugation, washed three times and then fractionated by SDS-PAGE followed by Western blotting with anti-flag (top panel) or anti-ASC (bottom panel) antibodies as indicated. (e) Purified preformed ASC pyroptosomes were incubated with 293 lysates containing WT flag-procaspase-1 (left panels) or purified WT flag-procaspase-1 (right panels) in the presence of the indicated potassium concentrations at $37^{\circ} \mathrm{C}$ for $1 \mathrm{~h}$. The total reaction mixtures were fractionated by SDS-PAGE followed by Western blotting with anti-flag (top panel) or anti-ASC (bottom panel) antibodies as indicated

completely soluble. Next we reduced potassium concentrations in the buffer gradually to $75 \mathrm{~mm}$ and incubated the samples at 37 or 4 C. As shown in Figure $8 \mathrm{a}$, incubation at $37^{\circ} \mathrm{C}$ in subphysiological concentrations of potassium induced the formation of ASC pyroptosomes, which pelleted at 5000 r.p.m. (lane 4). No ASC pyroptosomes were found in the sample that was incubated in $75 \mathrm{mM} \mathrm{KCl}$-containing buffer at $4^{\circ} \mathrm{C}$, (Figure $8 \mathrm{a}$, lane 5) indicating that incubation at $37^{\circ} \mathrm{C}$ is also an important factor in ASC oligomerization. The reconstituted ASC pyroptosomes have the same shape as the ASC pyroptosomes derived from THP-1 cells (not shown). Chemical crosslinking of the assembled ASC pyroptosomes with DSS, demonstrated that they are composed of oligomerized ASC dimers similar to those isolated from THP-1 cells (Figure 8b). Moreover, the reconstituted recombinant ASC pyroptosomes were able to activate procaspase-1 in a dose-dependent manner (Figure 8c), indicating that these pyroptosomes are functional. Collectively, these results indicate that subphysiological concentrations of potassium could directly induce ASC oligomerization in the absence of other cellular proteins.

\section{Discussion}

In this report, we addressed the role of the adapter protein ASC in the inflammatory cell death that has been recently termed pyroptosis. ${ }^{23,24}$ Although pyroptosis was first recognized in cells infected with intracellular pathogens, ${ }^{22-24}$ our results show that non-infectious agents like LPS, R837, MSU, SAT or lipopeptides can all induce pyroptosis in human THP-1 cells in the absence of an added ATP stimulus. Pyroptosis can also be induced in primary mouse bone marrow macrophages with LPS, but requires high concentrations of ATP as a second stimulus (Figure 3 and ref Mariathasan $S$ et al. ${ }^{14}$ and Perregaux $\mathrm{D}^{28}$ ). Recent studies suggested that activation of $\mathrm{P} 2 \mathrm{X} 7$ receptor by ATP causes the recruitment of pannexin-1 to the P2X7 receptor. ${ }^{36-38}$ The complex then form a large pore that mediates the delivery of bacterial products such as LPS into the cytosol, which is suggested to activate the cryopyrin inflammasome. ${ }^{39}$ However, it is still unclear why human monocytes such as THP-1 do not require ATP to induce caspase-1 activation in response to LPS and other pathogen and non-pathogen-associated molecules. Additionally, since high concentrations of ATP are not normally present in the extracellular milieu, the physiological significance of an 

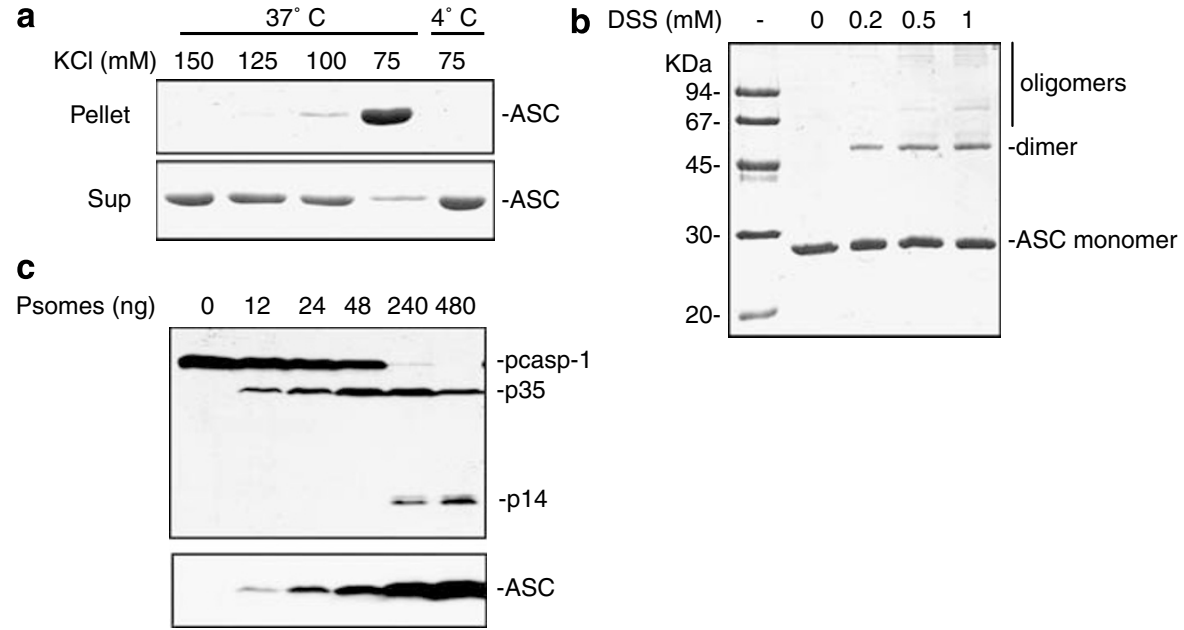

Figure 8 In vitro assembly of the ASC pyroptosome with purified ASC. (a) Purified recombinant ASC $(10 \mathrm{ng} / \mu \mathrm{l})$ was incubated at $37^{\circ} \mathrm{C}$ or at $4^{\circ} \mathrm{C}$ in the presence of increasing concentrations of $\mathrm{KCl}$ as indicated. The samples were centrifuged at 5000 r.p.m., and the resulting pellets (upper panel), which contain the assembled ASC pyroptosomes, and the remaining supernatants (lower panel) were then fractionated by SDS-PAGE followed by coomassie staining. (b) The assembled recombinant ASC pyroptosomes from (a) above were incubated with increasing concentrations of DSS for $30 \mathrm{~min}$ as indicated, and then fractionated by SDS-PAGE followed by Coomassie staining. (c) Increasing amounts of the assembled recombinant ASC pyroptosomes from (a) above were incubated with flag-procaspase- 1 for $1 \mathrm{~h}$ at $37^{\circ} \mathrm{C}$, and then fractionated by SDS-PAGE followed by Western blotting with anti-flag (top panel) or anti-ASC (bottom panel) antibodies as indicated

additional ATP stimulus to induce caspase-1 activation in mouse macrophages is questionable.

The pyroptotic process can be clearly observed in LPSstimulated THP-1 cells by live-cell time-lapse confocal microscopy (Supplementary movies 1-5). Using a GFPtagged ASC, we were able to track the pyroptotic cell death that occurred few minutes after formation of a large ASC oligomer that we termed here pyroptosome. This form of cell death is associated with extensive plasma membrane swelling and rupture, and is mediated by activation of caspase-1, as mouse macrophages deficient in caspase-1 (caspase $-1^{-1-}$ ) or HEK293T-ASC stable cells that are known to have no detectable caspase- 1 activity, do not exhibit these morphological features following formation of the ASC pyroptosome. Furthermore, inhibition of caspase-1 by zVADFMK, blocks LPS and other proinflammatory agents-induced pyroptotosis in THP-1 cells, but does not affect ASC pyroptosome formation. Our results thus clearly demonstrate that both IL-1 $\beta$ maturation and macrophage pyroptotic cell death are interlinked and are mediated by an ASC pyroptosome-induced caspase- 1 activation process. Pyroptosis is an important step in the inflammatory process because it serves as a mechanism by which activated macrophages rapidly release large amounts of their contents of activated cytokines such as IL-1 $\beta$ into the extracellular space to stimulate inflammation. Additionally, during infection with intracellular pathogens pyroptosis could play an important role to prevent pathogen overload by denying the pathogen from its host. ${ }^{21}$

In vitro incubation of THP-1 extracts at $37^{\circ} \mathrm{C}$ is believed to induce the assembly of the inflammasome. ${ }^{5}$ However, our results indicate that incubation of THP-1 extracts at $37^{\circ} \mathrm{C}$ induces formation of large ( $\sim 3 \mu \mathrm{m}$ in diameter) oligomeric ASC pyroptosomes similar to those observed in LPS-treated THP-1 cells. Therefore, it is not the NALP inflammasomes as claimed previously, ${ }^{5,10}$ but the ASC pyroptosomes that are largely responsible for the robust caspase-1 activation observed in $37^{\circ} \mathrm{C}$-stimulated THP-1 lysates. In support of this, $37^{\circ} \mathrm{C}$ stimulation of lysates from 293-ASC-caspase- 1 cells, which do not express any of the NALP proteins also resulted in robust caspase-1 activation (Figure 5a). Moreover, $37^{\circ} \mathrm{C}$ stimulation of purified ASC in a hypotonic buffer resulted in formation of functional pyroptosomes in the absence of other proteins (Figure 8).

Coomassie staining, mass spectroscopic and chemical crosslinking analyses of the purified pyroptosomes from THP1 cells provide compelling evidence that the mature pyroptosome is made of oligomerized ASC dimers that assemble into well-organized large crystal-like structures. The purified THP-1 pyroptosome contains procaspase- 1 and processed caspase-1, indicating that it is involved in the recruitment and activation of caspase- 1 . These observations suggest that the ASC pyroptosome is distinct from the conventional inflammasome which is believed to be made of an NLR family member (i.e., cryopyrin) in a complex with ASC and caspase-1. ${ }^{1,5,7,10}$ To our knowledge, this is the first cytosolic oligomer of this size that was ever isolated from cytosolic extracts by relatively low-speed centrifugation at 5000 r.p.m., except for structural complexes of the cell such as microtubules. The large size of the ASC pyroptosome is attributed to the high level of expression of endogenous ASC in monocytes and macrophages. Our estimates indicate that ASC is present in THP-1 cells at a concentration of $1.4 \mu \mathrm{g} / \mathrm{mg}$ total cytosolic proteins, compared to cryopyrin, which is present at a much lower level of $15 \mathrm{pg} / \mathrm{mg}$ total cytosolic proteins. Significantly, the ASC pyroptosome is a potent caspase- 1 activation platform due to its large size. Structurally caspase- 1 belongs to the initiator or apical caspase subfamily and requires dimerization by specific adapter proteins such as ASC and IPAF for activation. ${ }^{8,9,40}$ Since the ASC pyroptosome is made of oligomerized ASC dimers, this allows it to recruit, dimerize and activate almost all the endogenous procaspase-1 and still retain its potency to activate additional exogenous 
caspase-1, as demonstrated in vitro with purified ASC pyroptosoms from unstimulated or LPS-stimulated THP-1 cells (Figure $5 \mathrm{a}$ and $\mathrm{c}$ ). This unique property of the ASC pyroptosome truly makes it a potent death-inducing micromachine.

To elucidate the upstream molecular mechanism involved in the formation of the ASC pyroptosome and caspase-1 activation, we investigated the role of intracellular potassium depletion. Previous studies have established that there is a direct relationship between IL-1 $\beta$ secretion from macrophages and treatments that cause intracellular potassium depletion such as stimulation with bacterial toxins, or LPS coupled with ATP, ${ }^{14,27,28,34}$ but the mechanism by which subphysiological concentrations of potassium cause IL-1 $\beta$ generation remained unknown. Our results show that blocking potassium depletion by increasing extracellular potassium concentrations or blocking potassium channels inhibits formation of the ASC pyroptosome and subsequent cell death and secretion of IL-1 $\beta$ in LPS-treated THP-1 cells (Figure 6). We also observed that pore-forming toxins such as $S$. aureus $\alpha$-toxin or the potassium ionophore Nigericin, which are known to induce potassium depletion, are potent inducers of ASC pyroptosome formation in THP-1 cells (Figure $6 \mathrm{~d}$, and data not shown). Furthermore, non-specific plasma membrane permeabilization with digitonin, which has also been shown to augment potassium efflux ${ }^{41}$ was sufficient to induce pyroptosome formation in THP-1 cells. These results suggest that low potassium directly or indirectly affects formation of the ASC pyroptosome and may have additional effects on caspase-1 activation by the pyroptosome. A direct role of potassium depletion in the formation of the pyroptosome is supported by our findings that incubation of purified recombinant ASC at $37^{\circ} \mathrm{C}$ in a low potassium buffer in the absence of other cellular proteins is sufficient to induce formation of a functional pyroptosome. Importantly, low potassium concentrations were also required for recruitment and activation of caspase-1, since very little procaspase-1 recruitment and activation occurred at physiological potassium concentration. These results imply that potassium depletion is critical and is required for full caspase-1 activation not only because it facilitates the assembly of the pyroptosome, but also because it is critical for recruitment and robust activation of caspase-1 by the pyroptosome.

The assembly of the ASC pyroptosome is mediated by homotypic association of its PYD. This is supported by our finding that mutation of Lys26 to Ala in the PYD, which has been previously shown to inhibit self association of the isolated ASC PYD, ${ }^{33}$ also inhibited formation of the ASC pyroptosome. It is therefore likely that dimerization of the PYD of ASC is facilitated by subphysiological concentrations of potassium, possibly due to a decrease in intermolecular repulsion between ASC monomers at low potassium concentrations. Electrolytes such as sodium and potassium have been shown to cause strong intermolecular repulsion between protein monomers at physiological concentrations by helping in the formation of a water shell (hydration force) around the protein molecules. ${ }^{42}$ Thus a decrease in potassium concentration might lead to a decrease in the hydration force around the ASC monomers resulting in increased intermolecular attraction between the monomers, allowing the formation of dimers. Once formed, these dimers begin to oligomerize by a snowball effect, possibly driven by conformational changes in the dimer that enhance intermolecular attraction, culminating in the formation of one giant ASC pyroptosome in the cytoplasm.

The role of the adapter protein cryopyrin in the formation of the ASC pyroptosome is still elusive, because cryopyrindependent agents such as MSU and R83 $7^{13,15}$ and cryopyrinindependent agents such as LPS and the lipopeptides Pam3CSK4 and FSL- $1^{13}$ are all able to induce ASC pyroptosome formation in THP-1 cells in the absence of an added ATP stimulus. Our results also show that functional ASC pyroptosomes that were in vitro assembled from THP-1 lysates, or isolated from LPS-stimulated THP-1 cells do not contain any detectable amount of cryopyrin as determined by mass spectroscopy and Western blotting with a cryopyrinspecific antibody. These results are consistent with our findings that ASC is more abundantly expressed than cryopyrin in THP-1 cells ( $\sim 500$-fold molar excess). This makes it unlikely for the two proteins to be present in the pyroptosome at equimolar concentrations (as proposed for the cryopyrin inflammasome) since all ASC molecules assemble into one giant pyroptosome in each cell (Figure 2). In addition, functional ASC pyroptosome can be assembled with purified recombinant $A S C$, without cryopyrin, by mere incubation at $37^{\circ} \mathrm{C}$ in a buffer containing subphysiological concentrations of potassium (Figure 8). These findings indicate that cryopyrin, is not a pivotal component of the mature ASC pyroptosome and is not required for its activity. Therefore, the pyroptosome is a complex distinct from the cryopyrin inflammasome.

As to the possible role of cryopyrin in the formation of the pyroptosome, we speculate that it might function as an initial catalyst or an enhancer of ASC dimerization. For instance, oligomerization of cryopyrin by cryopyrin-dependent stimuli could result in the recruitment of monomeric ASC to the oligomerized cryopyrin. This interaction between cryopyrin and ASC might be enhanced by potassium depletion. The close proximity of the ASC molecules in this initial cryopyrinASC complex could induce formation of ASC dimers, which then dissociate from cryopyrin to form the nucleus upon which the ASC pyroptosome assembles. Further studies will be needed to address how cryopyrin is activated by diverse stimuli and its role in the formation of the ASC pyroptosome in response to cryopyrin-dependent stimuli.

Finally, a remaining question that needs to be addressed in future studies is whether activation of classical inflammasomes such as IPAF and NALP1 can also induce pyroptosis in macrophages independent of the ASC pyroptosome. Preliminary studies showed that IPAF forms an inflammasome complex with caspase-1 in response to infection of mouse macrophages with Salmonella. ${ }^{19}$ Interestingly, although ASCdeficient mouse macrophages contain IPAF, they showed delayed cell death and no detectable caspase- 1 activation in response to Salmonella infection. ${ }^{19}$ A possible explanation for these observations is that the IPAF concentration in cells might not be sufficient to induce robust caspase-1 activation, and so activation of the ASC pyroptosome is required to induce pyroptosis. A similar scenario might also be true for NALP1, which has been recently shown to form an inflammasome with caspase-1 in response to MDP. ${ }^{11}$ From these 
observations it is tempting to speculate that the classical inflammasomes such as IPAF, NALP1 and cryopyrin function upstream of ASC as conduits for controlled caspase-1 activation leading to limited inflammatory responses, but not cell death. In contrast, since ASC is present in macrophages at relatively high concentrations compared to IPAF, NALP1 and cryopyrin, direct or indirect activation of the ASC pyroptosome by the inflammasomes would result in more caspase- 1 activation, thereby causing cell death. The ASC pyroptosome function is perhaps required in a certain situation like infection with intracellular pathogens or extensive loss of intracellular potassium to eliminate the infected and damaged macrophages, and to mount a more robust inflammatory response such as that observed during septic shock. Supporting this distinct role of the ASC pyroptosome in cell death, recent investigations showed that IPAF and cryopyrindeficient macrophages are sensitive to LPS-induced septic shock, whereas ASC-deficient macrophages are completely resistant. ${ }^{17,19}$

\section{Materials and Methods}

Live-cell bioimaging. THP-1-ASC-GFP cells were seeded in $35 \mathrm{~mm}$ cover glass bottom culture dishes and then primed with PMA $(0.5 \mu \mathrm{M})$ for $3 \mathrm{~h}$ and allowed to attach for $24 \mathrm{~h}$. Time-lapse imaging was performed on an LSM 510 META Confocal Microscope System (Carl Zeiss) equipped with a temperature and $\mathrm{CO}_{2}$ controlled sample chamber for live-cell imaging. The GFP were excited with the $488 \mathrm{~nm}$ argon laser. The nuclear Hoecht 33342 stain was excited with the $405 \mathrm{~nm}$ diode laser. The mitotracker red was excited with the $543 \mathrm{~nm}$ He/Ne Laser 1. Ten minutes after crude LPS $(5 \mu \mathrm{g} / \mathrm{ml})$ stimulation, images from the GFP, Hoecht and mitotracker fluorescence signals were recorded simultaneously every $17.5 \mathrm{~s}$ for an additional $30 \mathrm{~min}$. Static bioimaging was done with wide-field fluorescence microscope or LSM 510 META confocal microscope.

ASC pyroptosome quantitation in live cells. THP-1-ASC-GFP cells were seeded in 12-well plates and then primed with PMA $(0.5 \mu \mathrm{M})$ for $3 \mathrm{~h}$. After priming the PMA containing medium was replaced with fresh medium and the cells were allowed to attach to the plates for $24 \mathrm{~h}$. The cells were pretreated with ZVAD FMK $(50 \mu \mathrm{M})$ for $30 \mathrm{~min}$ to prevent cell death, and then treated with different agents and observed by fluorescent microscopy at different periods of time after treatment. The number of cells containing ASC-GFP pyroptosomes were counted in several fields at the end of each period of time. The percentage of cells with ASC pyroptosomes was calculated by dividing the number of cells with ASC pyroptosomes over the total cells counted.

Treatment of THP-1 cells and assay of LDH and IL-1 $\beta$ secretion. PMA primed THP-1 cells were treated with ultrapure LPS (100 ng/ $\mathrm{ml}$ ) for $1 \mathrm{~h}$ to induce the synthesis of prolL- $1 \beta$ and then treated with different agents for different periods of time. The culture supernatants were collected at different time points and assayed for LDH with the CytoTox96 LDH-release kit (promega), and IL-1 $\beta$ by enzyme-linked immunosorbent assay (ELISA) (R\&D systems, Minneapolis, MN, USA), as described by the manufacturer's protocols.

Additional details on cells, purification and assembly of the ASC pyroptosome and reagents are given in the Supplementary Information.

Acknowledgements. We thank Dr. Junji Sagara for the ASC antibodies and Dr. Junying Yuan for the anti-mouse caspase-1 antibody. This work was supported by NIH grants AG14357 and CA78890 to ESA.

1. Martinon F, Tschopp J. NLRs join TLRs as innate sensors of pathogens. Trends Immunol 2005; 26: 447-454.

2. Ting JP, Kastner DL, Hoffman HM. Caterpillers, pyrin and hereditary immunological disorders. Nat Rev Immunol 2006; 6: 183-195.

3. Delbridge LM, O'Riordan MX. Innate recognition of intracellular bacteria. Curr Opin Immunol 2007; 19: 10-16.
4. Dinarello CA. Interleukin-1 beta, interleukin-18, and the interleukin-1 beta converting enzyme. Ann N Y Acad Sci 1998; 856: 1-11.

5. Martinon F, Burns K, Tschopp J. The inflammasome: a molecular platform triggering activation of inflammatory caspases and processing of prolL-beta. Mol Cell 2002; 10: 417-426.

6. Mariathasan S, Monack DM. Inflammasome adaptors and sensors: intracellular regulators of infection and inflammation. Nat Rev Immunol 2007; 7: 31-40.

7. Yu JW, Wu J, Zhang Z, Datta P, Ibrahimi I, Taniguchi S et al. Cryopyrin and pyrin activate caspase-1, but not NF-kappaB, via ASC oligomerization. Cell Death Differ 2006; 13: 236-249.

8. Srinivasula SM, Poyet JL, Razmara M, Datta P, Zhang Z, Alnemri ES. The PYRIN-CARD protein ASC is an activating adaptor for caspase-1. J Biol Chem 2002; 277: 21119-21122. Epub 2002 Apr 19

9. Poyet JL, Srinivasula SM, Tnani M, Razmara M, Fernandes-Alnemri T, Alnemri ES. Identification of Ipaf, a human caspase-1-activating protein related to Apaf-1. J Biol Chem 2001; 276: 28309-28313.

10. Agostini L, Martinon F, Burns K, McDermott MF, Hawkins PN, Tschopp J. NALP3 forms an IL-1beta-processing inflammasome with increased activity in Muckle-Wells autoinflammatory disorder. Immunity 2004; 20: 319-325.

11. Faustin B, Lartigue L, Bruey JM, Luciano F, Sergienko E, Bailly-Maitre B et al. Reconstituted NALP1 inflammasome reveals two-step mechanism of caspase-1 activation. Mol Cell 2007; 25: 713-724.

12. Boyden ED, Dietrich WF. Nalp1b controls mouse macrophage susceptibility to anthrax lethal toxin. Nat Genet 2006; 38: 240-244.

13. Kanneganti TD, Ozoren N, Body-Malapel M, Amer A, Park JH, Franchi L et al. Bacterial RNA and small antiviral compounds activate caspase-1 through cryopyrin/Nalp3. Nature 2006; 440: 233-236.

14. Mariathasan S, Weiss DS, Newton K, McBride J, O'Rourke K, Roose-Girma M et al. Cryopyrin activates the inflammasome in response to toxins and ATP. Nature 2006; 440: 228-232.

15. Martinon F, Petrilli V, Mayor A, Tardivel A, Tschopp J. Gout-associated uric acid crystals activate the NALP3 inflammasome. Nature 2006; 440: 237-241.

16. Kanneganti TD, Body-Malapel M, Amer A, Park JH, Whitfield J, Taraporewala ZF et al. Critical role for cryopyrin/Nalp3 in activation of caspase-1 in response to viral infection and double-stranded RNA. J Biol Chem 2006; 281: 36560-36568.

17. Sutterwala FS, Ogura Y, Szczepanik M, Lara-Tejero M, Lichtenberger GS, Grant EP et al. Critical role for NALP3/CIAS1/Cryopyrin in innate and adaptive immunity through its regulation of caspase-1. Immunity 2006; 24: 317-327.

18. Masumoto J, Taniguchi S, Ayukawa K, Sarvotham H, Kishino T, Niikawa N et al. ASC, a novel 22-kDa protein, aggregates during apoptosis of human promyelocytic leukemia HL-60 cells. J Biol Chem 1999; 274: 33835-33838.

19. Mariathasan S, Newton K, Monack DM, Vucic D, French DM, Lee WP et al. Differential activation of the inflammasome by caspase-1 adaptors ASC and Ipaf. Nature 2004; 430 : 213-218. Epub 2004 Jun 09

20. Yamamoto M, Yaginuma K, Tsutsui H, Sagara J, Guan X, Seki E et al. ASC is essential for LPS-induced activation of procaspase-1 independently of TLR-associated signal adaptor molecules. Genes Cells 2004; 9: 1055-1067.

21. Mariathasan S, Weiss DS, Dixit VM, Monack DM. Innate immunity against Francisella tularensis is dependent on the ASC/caspase-1 axis. J Exp Med 2005; 202: 1043-1049.

22. Swanson MS, Molofsky AB. Autophagy and inflammatory cell death, partners of innate immunity. Autophagy 2005; 1: 174-176.

23. Fink SL, Cookson BT. Caspase-1-dependent pore formation during pyroptosis leads to osmotic lysis of infected host macrophages. Cell Microbiol 2006; 8: 1812-1825.

24. Fink SL, Cookson BT. Apoptosis, pyroptosis, and necrosis: mechanistic description of dead and dying eukaryotic cells. Infect Immun 2005; 73: 1907-1916.

25. Cookson BT, Brennan MA. Pro-inflammatory programmed cell death. Trends Microbiol 2001; 9: 113-114.

26. Monack DM, Navarre WW, Falkow S. Salmonella-induced macrophage death: the role of caspase-1 in death and inflammation. Microbes Infect 2001; 3: 1201-1212.

27. Warny M, Kelly CP. Monocytic cell necrosis is mediated by potassium depletion and caspase-like proteases. Am J Physiol 1999; 276: C717-C724.

28. Perregaux D, Gabel CA. Interleukin-1 beta maturation and release in response to ATP and nigericin. Evidence that potassium depletion mediated by these agents is a necessary and common feature of their activity. J Biol Chem 1994; 269: 15195-15203.

29. Aliprantis AO, Yang RB, Weiss DS, Godowski P, Zychlinsky A. The apoptotic signaling pathway activated by Toll-like receptor-2. Embo J 2000; 19: 3325-3336.

30. Aliprantis AO, Yang RB, Mark MR, Suggett S, Devaux B, Radolf JD et al. Cell activation and apoptosis by bacterial lipoproteins through toll-like receptor-2. Science 1999; 285: 736-739.

31. Aliprantis AO, Weiss DS, Radolf JD, Zychlinsky A. Release of Toll-like receptor-2-activating bacterial lipoproteins in Shigella flexneri culture supernatants. Infect Immun 2001; 69: 6248-6255.

32. Aliprantis AO, Weiss DS, Zychlinsky A. Toll-like receptor-2 transduces signals for NFkappa B activation, apoptosis and reactive oxygen species production. J Endotoxin Res 2001; 7: 287-291.

33. Moriya M, Taniguchi S, Wu P, Liepinsh E, Otting G, Sagara J. Role of charged and hydrophobic residues in the oligomerization of the PYRIN domain of ASC. Biochemistry 2005; 44: 575-583. 
34. Walev I, Reske K, Palmer M, Valeva A, Bhakdi S. Potassium-inhibited processing of IL-1 beta in human monocytes. EMBO J 1995; 14: 1607-1614.

35. Cain K, Langlais C, Sun XM, Brown DG, Cohen GM. Physiological concentrations of K+ inhibit cytochrome c-dependent formation of the apoptosome. J Biol Chem 2001; 276: 41985-41990.

36. Locovei S, Scemes E, Qiu F, Spray DC, Dahl G. Pannexin1 is part of the pore forming unit of the P2X(7) receptor death complex. FEBS Lett 2007; 581: 483-488.

37. Pelegrin $P$, Surprenant $A$. Pannexin-1 couples to maitotoxin- and nigericin-induced interleukin-1beta release through a dye uptake-independent pathway. J Biol Chem 2007; 282: 2386-2394.

38. Pelegrin $P$, Surprenant A. Pannexin-1 mediates large pore formation and interleukin-1beta release by the ATP-gated P2X7 receptor. EMBO J 2006; 25: 5071-5082.
39. Kanneganti TD, Lamkanfi M, Kim YG, Chen G, Park JH, Franchi L et al. Pannexin-1-mediated recognition of bacterial molecules activates the cryopyrin inflammasome independent of toll-like receptor signaling. Immunity 2007; 26: 433-443.

40. Martinon F, Tschopp J. Inflammatory caspases: linking an intracellular innate immune system to autoinflammatory diseases. Cell 2004; 117: 561-574.

41. Andersson B, Behnam-Motlagh P, Henriksson R, Grankvist K. Pharmacological modulation of lung cancer cells for potassium ion depletion. Anticancer Res 2005; 25: 2609-2616.

42. Petsev DN, Thomas BR, Yau S, Vekilov PG. Interactions and aggregation of apoferritin molecules in solution: effects of added electrolytes. Biophys $J$ 2000; 78 : 2060-2069.

Supplementary Information accompanies the paper on Cell Death and Differentiation website (http://www.nature.com/cdd) 